\title{
Prym Varieties and the Schottky Problem
}

\author{
Arnaud Beauville \\ Départment de Mathématiques, Université d'Angers, F-49000 Angers, France
}

\section{Introduction}

The Schottky problem is the problem of characterizing Jacobian varieties among all abelian varieties.

More precisely, let:

$$
\mathscr{A}_{\mathrm{g}}=H_{\mathrm{g}} / \operatorname{Sp}(\mathrm{Z}, 2 \mathrm{~g})
$$

be the moduli space of principally polarized abelian varieties of dimension $g$, $J_{g} \subset \mathscr{A}_{g}$ the locus of Jacobians. The problem is to find explicit equations for $J_{g}$ (or rather its closure $\bar{J}_{g}$ ) in $\mathscr{A}_{g}$.

In their beautiful paper [A-M], Andreotti and Mayer prove that $\bar{J}_{\mathrm{g}}$ is an irreducible component of the locus $N_{g_{-4}}$ of principally polarized abelian varieties $(A, \Theta)$ with $\operatorname{dim} \operatorname{Sing} \Theta \geqq g-4$. Then they give a procedure to write "explicit" equations for $N_{8-4}$.

There is no hope that $\bar{J}_{g}$ be equal to $N_{g-4}$ : already in genus 4 , there is at least one other component, namely the divisor $\theta_{\text {null }}$ of principally polarized abelian varieties with one vanishing theta-null (i.e. such that $\operatorname{Sing} \Theta$ contains a point of order 2). Our aim is to prove the following:

Theorem. a) $N_{0}=\bar{J}_{4} \cup \theta_{\text {null }}$.

b) The divisor $\theta_{\text {null }}$ is irreducible.

In genus 5, the locus $N_{1} \subset \mathscr{A}_{5}$ has already many components. However, we prove that $\bar{J}_{5}$ is the only component of $N_{1}$ not contained in $\theta_{\text {null }}$.

The proofs use the fact that a generic principally polarized abelian variety of dimension 4 or 5 is a Prym variety. In [M 2], Mumford gives a complete list of all Prym varieties with $\operatorname{dim} \operatorname{Sing} \Theta \geqq g-4$. If every principally polarized abelian variety (of dimension 4 or 5) were a Prym variety, the results would follow at once; however we see immediately from Mumford's list that the product of an elliptic curve and a non-hyperelliptic Jacobian-for instance-is not a Prym variety in the classical sense. 
Thus the main ingredient of the proofs is the construction of the generalized Prym varieties which appear in the closure of the locus of ordinary Prym varieties in $\mathscr{A}_{\mathrm{g}}$. It turns out that the whole theory of Prym varieties, as developped in [M 1] and [M 2], extends to certain coverings of curves with ordinary double points. These generalized Prym varieties were known in the case of one ordinary double point (see [M 2] and (3.6) below); in general, they naturally appear as intermediate Jacobians of certain non-singular varieties, for instance the intersection of three quadrics in $\mathbf{P}^{2 n}(n \geqq 2)$.

After some preliminary results $(\S 1$ and 2$)$, we define the generalized Prym varieties in $\S 3$. In $\S 4$, we give a list of all the generalized Prym varieties with dim Sing $\Theta \geqq g-4$. The method is that of [M 2], but there are some technical difficulties due to reducible curves. It should be noted that the proof of the result for Prym varieties of dimension 4 and 5 (the only one to be used in this paper) is considerably simpler; in particular, the hardest part of Lemma 4.9. (from (4.9.4) on) is not needed. However, we have insisted on giving a general proof because of the application to intermediate Jacobians: thus Theorem 4.10 implies for instance that every smooth intersection of three quadrics in $\mathbf{P}^{6}$ is non-rational.

In $\S 5$ and 6, we prove that the locus of generalized Prym varieties is closed in $\mathscr{A}_{g}$; this follows from the work of Deligne and Mumford on compactification of the moduli space of curves. In $\S 7$ and 8 we apply these results to principally polarized abelian varieties of dimension 4 and 5 .

Most of our results are actually valid over an algebraically closed field of any characteristic different from two. In particular, we obtain as a consequence the irreducibility of the moduli space of principally polarized abelian varieties of dimension 4 or 5 , over a field of characteristic $p \neq 2^{1}$.

I am heavily indebted to $\mathrm{H}$. Clemens for his continual encouragement. I wish to thank Columbia University for its hospitality during the preparation of this paper.

\section{Terminology and Notation}

Throughout this paper we fix an algebraically closed field $k$ of characteristic $\neq 2$; all varieties considered are defined over $k$. By a point of a variety we mean a point rational over $k$.

A curve is a one-dimensional variety over $k$ (that is, a one-dimensional reduced scheme of finite type over $k$ ). The genus $p_{a}(C)$ of a curve $C$ is defined by:

$$
p_{a}(C)=1-\chi\left(\mathcal{O}_{C}\right)
$$

Let $C_{1}, \ldots, C_{c}$ be the irreducible components of $C$. For any vector bundle $E$ on $C$, the multidegree $\operatorname{deg}(E)=\left(r_{1} \ldots r_{r}\right)$ is defined by $r_{i}=\operatorname{deg} E_{\mid C_{i}}$ and the degree of $E$ by $\operatorname{deg}(E)=\sum r_{i}$. For any coherent sheaf $F$ on $C$, we write:

$$
\operatorname{dim} H^{0}(C, F)=h^{0}(C, F)
$$

(or $h^{0}(F)$ if there is no ambiguity on $C$ ).

\footnotetext{
1 I am grateful to F. Oort for pointing out to me that this fact was not known, and that it should be a consequence of the results contained in this paper
} 


\section{Theta-Characteristics on a Singular Curve}

In this section we indicate how to modify the proofs in [M 1] to get the corresponding results for curves with arbitrary singularities.

\section{(1.1) Theorem. Let}

(i) $\pi: \mathscr{X} \rightarrow S$ be a proper, flat family of curves.

(ii) $\mathscr{E}$ a coherent $\mathcal{O}_{\mathscr{X}}$-Module, flat over $S$, such that for all $s \in S$ the induced sheaf $\mathscr{E}_{s}$ is torsion-free of rank $r$.

(iii) $Q: \mathscr{E} \rightarrow \omega_{\mathscr{X} / S}$ a non-degenerate quadratic form.

Then the function $s \mapsto \operatorname{dim} H^{\circ}\left(\mathscr{X}_{s}, \mathscr{E}_{s}\right) \bmod 2$ is constant on connected components of $S$.

Here $\omega_{\mathscr{T} / S}$ is the relative dualizing sheaf $f^{!} \mathcal{O}_{S}([\mathrm{H}])$, that is, a sheaf whose restriction to each fibre $\mathscr{X}_{s}$ is the dualizing sheaf $\omega_{\mathscr{X}_{s}}$. By (iii), we mean that $Q$ induces an isomorphism $\mathscr{E}_{s} \stackrel{\sim}{\longrightarrow} \underline{\operatorname{Hom}}_{\mathscr{X}_{s}}\left(\mathscr{E}_{s}, \omega_{\mathscr{X}_{s}}\right)$ for each $s$.

1) Define $\mathfrak{a}=\sum_{i=1}^{N} P_{i}$ where the $P_{i}$ are non-singular points of $\mathscr{X}_{s}$. Since $E=\mathscr{E}_{s}$ is locally free outside the singular locus, one gets as in $[\mathrm{M} 1]: \Gamma(E)=\Gamma(E(\mathrm{a}))$ $\cap \Gamma(E / E(-a))$ in $\Gamma(E(a) / E(-a))$.

2) Use Grothendieck duality instead of Serre duality: since $\operatorname{Ext}_{\mathscr{T}_{s}}^{1}\left(E, \omega_{x_{s}}\right)=0$ by local duality, one still gets $\operatorname{dim} W_{1}=\operatorname{dim} W_{2}=N r, \operatorname{dim} V=2 \mathrm{Nr}$.

3) Replace ordinary residue by generalized residue ([A-K]). The function on $E(\mathfrak{a}) / E(-\mathfrak{a})$ given by $q(\bar{a})=\sum_{i=1}^{N} \operatorname{Res}_{p_{i}} Q\left(a_{i}\right)$ still defines a non-degenerate quadratic form on $V$.

The theorem follows as in [M1].

Before stating the following corollary, we fix some notation: we denote by $J_{2}$ the set of line bundles $L$ on $C$ such that $L^{2} \cong \mathcal{O}_{C}$; we define the pairing $e_{2}$ on $J_{2}$ by:

$$
e_{2}(\alpha, \beta)=e_{2, N}\left(f^{*} \alpha, f^{*} \beta\right)
$$

where $f: N \rightarrow C$ is the normalization of $C$, and $e_{2, N}:(J N)_{2} \rightarrow\{ \pm 1\}$ is the pairing induced on the group of points of order 2 by the Riemann form of $J N$ ([M3]); we could as well define $e_{2}$ directly as for abelian varieties, or by the cup product on $H^{1}(C, \mathrm{Z} /(2))$.

(1.2) Corollary. Let $C$ be a curve, $L_{0}$ a "theta-characteristic" on $C$ (a torsion free, rank one $\mathcal{O}_{C}$-module such that $\left.L_{0} \cong \underline{\operatorname{Hom}}\left(L_{0}, \omega_{C}\right)\right)$. Then the map from $J_{2}$ to $\mathbf{Z} /(2)$ defined by

$$
L \mapsto h^{0}\left(L_{0} \otimes L\right)+h^{0}\left(L_{0}\right) \quad(\bmod 2)
$$

is a quadratic form on $J_{2}$ whose associated bilinear form is $e_{2}$.

Proof. Let $L, M$ be two line bundles on $C$ such that $L^{2} \cong M^{2} \cong \mathcal{O}_{C}$. We want to prove:

$$
h^{0}\left(L_{0}\right)+h^{0}\left(L_{0} \otimes L\right)+h^{0}\left(L_{0} \otimes M\right)+h^{0}\left(L_{0} \otimes L \otimes M\right) \equiv \ln e_{2}(L, M)
$$


where $\ln (1)=0, \ln (-1)=1$. One defines a quaternion algebra structure on $A=\mathcal{O}_{C}+L+M+L \otimes M$ as in [M1]. Then $A$ is isomorphic to Hom $(E, E)$ for some rank 2 vector bundle $E$ on $C$ : and since $f^{*} A \cong$ Hom $\left(f^{*} E, f^{*} E\right)$, Lemma 2 in $[\mathrm{M} 1]$ gives:

$$
\operatorname{deg} E \equiv \ln e_{2}(L, M) \quad \bmod 2 .
$$

Now by Lemma 1.3 below the set of vector bundles of given rank and multidegree is connected, hence by the theorem:

$$
h^{0}\left(L_{0} \otimes A\right) \equiv h^{0}\left(L_{0} \otimes \underline{\operatorname{Hom}}\left(E^{\prime}, E^{\prime}\right)\right) \quad \bmod 2
$$

for any rank 2 vector bundle $E^{\prime}$ with $\operatorname{deg} E^{\prime}=\operatorname{deg} E$. We pick a line bundle $F$ on $C$ with $\underline{\operatorname{deg} F}=\underline{\operatorname{deg}} E$, and take $E^{\prime}=\mathscr{O}_{C} \overline{\oplus F}$. Then:

$$
\begin{aligned}
h^{0}\left(L_{0} \otimes \underline{\operatorname{Hom}}\left(E^{\prime}, E^{\prime}\right)\right) & =2 h^{0}\left(L_{0}\right)+h^{0}\left(L_{0} \otimes F\right)+h^{0}\left(L_{0} \otimes F^{-1}\right) \\
& \equiv h^{0}\left(L_{0} \otimes F\right)-h^{0}\left(L_{0} \otimes F^{-1}\right) \quad \bmod 2 \\
& =\chi\left(L_{0} \otimes F\right) \quad \text { by Grothendieck duality } \\
& =\operatorname{deg} F \quad \text { by Riemann-Roch } \\
& =\ln e_{2}(L, M) .
\end{aligned}
$$

(1.3) Lemma. Given integers $r \geqq 1 ; d_{1}, \ldots, d_{c}$, there exists an irreducible variety $S$ and $a$ vector bundle $\mathscr{E}$ on $C \times S$ of rank $r$ and multidegree $\left(d_{1}, \ldots, d_{c}\right)$ such that any vector bundle of rank $r$ and multidegree $\left(d_{1}, \ldots, d_{c}\right)$ on $C$ is isomorphic to $\mathscr{E}_{\mid C \times\{s\}}$ for some $s \in S$.

Proof. The lemma is well-known if $C$ is non-singular ([S]). If $C$ is singular, define the skyscraper sheaf $\delta$ by the exact sequence:

$$
0 \rightarrow \mathcal{O}_{C} \rightarrow f_{*} \mathcal{O}_{N} \rightarrow \delta \rightarrow 0 .
$$

Let $E$ be a rank $r$ vector bundle on $C$. Choosing an isomorphism $E \stackrel{\sim}{\rightarrow}$ 舫 near the singular points, we get:

$$
0 \rightarrow E \rightarrow f_{*} f^{*} E \rightarrow \delta^{r} \rightarrow 0 .
$$

Thus any vector bundle $E$ on $C$ can be given by a vector bundle $F=f^{*} E$ on $N$, plus a "descent data" morphism $h: f_{*} F \rightarrow \delta^{r}$ which must be surjective with locally free kernel.

Now let $T$ be an irreducible variety parametrizing all vector bundles of rank $r$ and multidegree $\left(d_{1}, \ldots, d_{c}\right)$ on $N, \mathscr{E}$ the corresponding vector bundle on $N \times T$, $p, q$ the projections from $C \times T$ onto $C, T$.

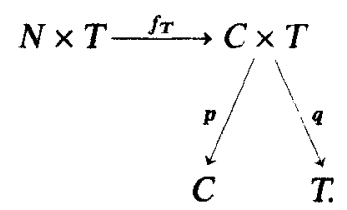


The sheaf $H=q_{*} \underline{\operatorname{Hom}}\left(f_{T_{*}} \mathscr{E}, p^{*} \delta^{r}\right)$ is locally free on $T$ (since locally over $T$ we may replace $\mathscr{E}$ by $\mathcal{O}_{C \times T}^{r}$; then $H$ becomes isomorphic to $q_{*} p^{*} G$, with $G=$ $\underline{\text { Hom }}_{C}\left(f_{*} \mathcal{O}_{N}^{r}, \delta^{r}\right)$, but $q_{*} p^{*} G=\mathcal{O}_{T} \otimes_{k} H^{0}(C, G)$ is a free $\mathcal{O}_{T}$-module). We denote by $S_{1}$ the associated vector bundle $\left(S_{1}=\mathbf{V}(\breve{H})\right.$ in EGA notation), by $k: C \times S_{1} \rightarrow$ $C \times T$ the projection; on $C \times S_{1}$ there is a canonical morphism:

$$
h: k^{*} f_{T^{*}} \mathscr{E} \rightarrow k^{*} p^{*} \delta^{r}
$$

such that a point $s$ in $S_{1}$ is given by a point $t \in T$, together with a morphism $h_{\mid C \times(s)}$ : $f_{*}\left(\mathscr{E}_{\mid N \times\{t\}}\right) \rightarrow \delta^{r}$. We take the open set $S$ in $S_{1}$ consisting of points $s \in S$ such that $h_{\mid C \times\{s\}}$ is surjective with locally free kernel. $S$ is irreducible, and by what we have seen, $S$ together with the vector bundle $\operatorname{Ker} h$ on $C \times S$ give a complete family of vector bundles on $C$.

\section{Theta Divisor of a Generalized Jacobian}

We consider a connected curve $C$ of genus $g$, with only ordinary double points. We denote by $J C$ the generalized Jacobian of $C$; recall that $J C$ is a smooth commutative algebraic group, and the points of $J C$ can be naturally identified with isomorphism classes of line bundles on $C$ of multidegree $(0, \ldots, 0)$. The normalization $f: N \rightarrow C$ induces an epimorphism $f^{*}: J C \rightarrow J N$, whose kernel is a torus (i.e. a multiplicative group $\left.\left(\mathbf{G}_{m}\right)^{t}\right)$. For any line bundle $L$ of degree $g-1$ on $C$, we define $\Theta_{L}$ as the locus of line bundles $M$ in $J C$ such that: $h^{0}(L \otimes M) \geqq 1$. We denote by $\Theta^{\prime}$ the theta divisor on $J N$ (defined up to translation).

Our aim is to prove that $\Theta_{L}$ is a divisor, algebraically equivalent to $\left(f^{*}\right)^{-1}\left(\Theta^{\prime}\right)$. However, if $C$ is reducible, this will be true only for a good choice of $\operatorname{deg}(L)$. To deal with this case, we associate to the curve $C$ a graph $\Gamma$ :

The set of vertices of $\Gamma$ is the set $\left\{C_{1}, \ldots, C_{c}\right\}$ of irreducible components of $C$; an edge between two vertices $C_{i}, C_{j}$ corresponds to a point of $C_{i} \cap C_{j}$.

(2.1) Lemma. Let $\underline{d}=\left(d_{1}, \ldots, d_{c}\right)$ be a multidegree such that $\sum d_{i}=g-1$. The following conditions are equivalent:

(i) There exists a line bundle $L$ on $C$, of multidegree $\underline{d}$, with $h^{0}(L)=0$.

(ii) Given line bundles $L_{0}$ on $C$, and $L_{1}$ on $N$ such that:

$\underline{\operatorname{deg}}\left(L_{0}\right)=\underline{d} ; \operatorname{deg}\left(L_{1}\right)=\frac{1}{2} \operatorname{deg}\left(\omega_{N}\right)$, there exists an irreducible non-singular variety $S$, a coherent sheaf $\mathscr{L}$ on $C \times S$, flat over $S$, and two points $s_{0}, s_{1} \in S$ such that: - $\mathscr{L}_{s}=\mathscr{L}_{\mid C \times\{s\}}$ is torsion-free of rank one, for each $s$ in $S$;

- $\mathscr{L}_{s_{0}} \cong L_{0}$

$-\mathscr{L}_{s_{1}} \cong f_{*}\left(L_{1}\right)$.

(iii) The graph $\Gamma$ can be oriented in such a way that, if $k_{i}$ denotes the number of edges starting from $C_{i}$, one has:

$$
d_{i}=p_{a}\left(C_{i}\right)-1+k_{i} \quad(i=1, \ldots, c) .
$$

Assume moreover that the restriction of $\omega_{C}$ to $C_{i}$ has even degree for each $i$; then the multidegree $\underline{d}=\frac{1}{2} \underline{\operatorname{deg}}\left(\omega_{c}\right)$ satisfies conditions (i) to (iii). 
Proof. (iii) $\Rightarrow$ (ii). We use the same construction (and notation) as in Lemma 1.3. Here we take for $T$ the variety of line bundles $L$ on $N$ with $\operatorname{deg} L=\underline{d}$, and for $\mathscr{E}$ a Poincaré line bundle on $N \times T$; we define $S_{1}$ as in Lemma $\overline{1.3}$ and denote by $S_{0}$ the open set in $S_{1}$ consisting of points $s$ such that $h_{\mid C \times\{s\}}$ is surjective; so a point $s$ of $S_{0}$ corresponds to a line bundle $L$ on $N$ (of multidegree $\underline{d}$ ), together with a surjective morphism on $C: h_{s}: f_{*} L \rightarrow \delta$. Let $z$ be a singular point, $f^{-1}(\{z\})=\{x, y\}$, $U$ a neighborhood of $z$ in $C$ which contains no other singular points and such that $L \cong \mathcal{O}_{N}$ on $f^{-1}(U)$. Choosing a generator of $L$ on $f^{-1}(U)$, one checks easily that $h_{s}$ is given by:

$$
h_{s}(t)=\alpha t(x)+\beta t(y) \quad \text { with } \alpha, \beta \in k .
$$

If $\alpha$ and $\beta \neq 0, \operatorname{Ker} h_{s}$ is an invertible sheaf on $U$; if for instance $\alpha \neq 0, \beta=0$, then Ker $h_{s}$ is isomorphic on $U$ to $f_{*}\left(\mathcal{O}_{N}(-x)\right)$.

Define $\mathscr{L}=\operatorname{Ker} h$ on $S_{0}$. Since $k^{*} \mathscr{E}$ and $k^{*} p^{*} \delta$ are flat on $S_{0}$ and $h$ is surjective, $\mathscr{L}$ is flat on $S_{0}$. Let $s$ be a point in $S_{0}$. If $h_{s}$ is such that $\alpha$ and $\beta \neq 0$ at each singular point, then $\mathscr{L}_{s}=\mathscr{L}_{\mid C \times\{s\}}$ is an invertible sheaf of multidegree $\underline{d}$, and all the invertible sheaves of multidegree $\underline{d}$ are obtained that way. On the other hand, if we choose $s$ such that $\alpha . \beta=0$ at every singular point, then

$$
\mathscr{L}_{s}=f_{*}\left(L\left(-\sum_{i} x_{i}\right)\right)
$$

where $\left\{f\left(x_{1}\right), \ldots, f\left(x_{m}\right)\right\}$ is the set of singular points in $C$.

To achieve the proof that (iii) implies (ii), we must show that we can choose one point $x_{i} \in N$ above each double point $z_{i}$ of $C$ in such a way that: $2 \operatorname{deg} L\left(-\sum_{i} x_{i}\right)$ $=\operatorname{deg} \omega_{N}$. If $z_{i}$ belongs to only one component of $C$, we choose $x_{i}$ arbitrarily (among the two points of $f^{-1}\left(z_{i}\right)$ ). Suppose that $z_{i}$ belongs to two components. We consider the graph $\Gamma$; we assume it is oriented so that property (iii) holds. The point $z_{i}$ corresponds to an edge of $\Gamma$, and we choose the point $x_{i} \in f^{-1}\left(z_{i}\right)$ which lies in the component corresponding to the starting point of the edge.

For $1 \leqq j \leqq c$, let $l_{j}$ be the number of double points of $C$ which belong to $C_{j}$ and not to $C_{k}$ for $k \neq j$. Then:

$\operatorname{deg} L\left(-\sum_{i} x_{i}\right)_{\mid C_{j}}=d_{j}-k_{j}-l_{j}=p_{a}\left(C_{j}\right)-l_{j}-1=p_{a}\left(N_{j}\right)-1$, where $N_{j}$ is the component of $N$ which dominates $C_{j}$. Hence, $2 \operatorname{deg} L\left(-\sum_{i} x_{i}\right)=\underline{\operatorname{deg}} \omega_{N}$, so we have
proved that (iii) implies (ii).

(ii) $\Rightarrow$ (i): Assume that property (ii) holds. We can choose $L_{1}$ such that $h^{0}\left(N, L_{1}\right)$ $=0$; then there is a neighbourhood $U_{1}$ of $s_{1}$ in $S$ such that $h^{0}\left(C, \mathscr{L}_{s}\right)=0$ for $s \in U_{1}$. On the other hand there is a neighbourhood $U_{0}$ of $s_{0}$ in $S$ such that $\mathscr{L}_{t}$ is an invertible sheaf of multidegree $d$ when $t \in U_{0}$. If $u \in U_{0} \cap U_{1}$, the invertible sheaf $L=\mathscr{L}_{u}$ satisfies condition (i).

(i) $\Rightarrow$ (iii): We prove this by induction on the number $m$ of edges of $\Gamma$. If $m=0$, there is nothing to prove. If $m>0$, let us choose an edge of $\Gamma$, i.e. a point $s$ of $C_{i} \cap C_{j}$ $(i \neq j)$. Let $f_{s}: N_{s} \rightarrow C$ be the normalization of $C$ at the point $s$. We get as before an exact sequence:

$$
0 \rightarrow L \rightarrow\left(f_{s}\right)_{*} f_{s}^{*} L \stackrel{h_{s}}{\longrightarrow} \delta \rightarrow 0, \quad \text { with } \delta=k(s) .
$$


Since $h^{0}(L)=0$ by hypothesis and $h^{1}(L)=0$ by Riemann-Roch, the mapping:

$$
h_{s}: H^{0}\left(N_{s}, f_{s}^{*} L\right) \rightarrow \delta
$$

is an isomorphism. Therefore $H^{0}\left(N_{s}, f_{s}^{*} L\right)$ is generated by a section $t$ such that: $h_{s}(t) \neq 0$. If $f^{-1}(s)=\left\{s_{i}, s_{j}\right\}$, we have seen that: $h_{s}(t)=\alpha t\left(s_{i}\right)+\beta t\left(s_{j}\right)$, with $\alpha$ and $\beta \neq 0$. It follows that $t$ cannot vanish identically both on $C_{i}$ and $C_{j}$. Suppose that $t$ does not vanish identically on $C_{i}$; then if $x$ is a generic point of $C_{i}$, the sheaf $L^{\prime}=f_{s}^{*} L(-x)$ on $N_{s}$ verifies $h^{0}\left(L^{\prime}\right)=0$. If $N_{s}$ is connected, one has $\operatorname{deg}\left(L^{\prime}\right)=p_{a}\left(N_{s}\right)-1$, so that one can apply the induction hypothesis to $L$; if $N_{s}$ has two connected components $N_{1}, N_{2}$, one checks that: $\operatorname{deg} L_{\mid N_{i}}^{\prime}=p_{a}\left(N_{i}\right)-1(i=1,2)$, so that we can apply the induction hypothesis to $L_{\mid N_{1}}^{\prime}$ and $L_{\mid N_{2}}^{\prime}$. In both cases, if $e_{s}$ denotes the edge of $\Gamma$ corresponding to $s$, we can find an orientation of $\Gamma-\left\{e_{s}\right\}$ such that (iii) holds (with respect to $\operatorname{deg}\left(L^{\prime}\right)$ ); then we orient the edge $e_{s}$ from $C_{i}$ to $C_{j}$. It is immediate that the orientation obtained for $\Gamma$ satisfies (iii).

The last assertion of the lemma follows from Euler's graph theorem: a graph $\Gamma$, such that the number of edges passing through each vertex is even, can be oriented in such a way that at each vertex $p$, the number of edges starting from $p$ equals the number of edges abutting to $p$.

Recall that we denote by $\Theta^{\prime}$ a theta divisor on $J N$, and by $\Theta_{L}$ the locus of line bundles $M$ in $J C$ such that $h^{0}(L \otimes M) \geqq 1$.

(2.2) Proposition. Let $L$ be a line bundle on $C$ whose multidegree satisfies the equivalent conditions of Lemma 2.1 (for instance $2 \operatorname{deg}(L)=\operatorname{deg}\left(\omega_{C}\right)$ ). Then $\Theta_{L}$ is a divisor, algebraically equivalent to $\left(f^{*}\right)^{-1}\left(\Theta^{\prime}\right)$.

Proof. Choose $S, \mathscr{L}, s_{0}, s_{1}$ as in condition (ii) of the lemma, with $L=L_{0}$. We want to construct a divisor $Z$ in $J C \times S$, flat over $S$, such that for each $s$ in $S, Z_{s}$ is the locus of line bundles $L$ in $J C$ with $h^{0}\left(\mathscr{L}_{s} \otimes L\right) \geqq 1$. We use Kempfs construction (see [Sz]). Let $\mathscr{P}$ be a Poincare bundle on $C \times J C, p, q, r, m$ the projections from $C \times J C \times S$ onto $C \times S, J C \times S, C \times J C, C$. We put $\mathscr{F}=r^{*} \mathscr{P} \otimes p^{*} \mathscr{L}$. Let $s \in S$, $\alpha \in J C$ (corresponding to a line bundle $L_{\alpha}$ on $C$ ); one has by definition:

$$
\mathscr{F}_{\mid C \times\{\alpha\} \times\{s\}}=\mathscr{L}_{s} \otimes L_{\alpha}
$$

Let us choose $g$ non-singular points $x_{1}, \ldots, x_{\mathrm{g}}$ on $C$ such that if $D=\sum x_{i}$, one has $\operatorname{deg} D_{\mid C_{j}} \geqq d_{j}$ for each $j$. Since $\mathscr{L}$ is invertible around $\left\{x_{i}\right\} \times S$, we get an exact sequence:

$$
0 \rightarrow \mathscr{F} \otimes m^{*} \mathcal{O}_{C}(-D) \rightarrow \mathscr{F} \rightarrow \mathscr{F} \otimes m^{*} \mathcal{O}_{D} \rightarrow 0
$$

Apply $q_{*}$ to this exact sequence; we claim that:

$-q_{*}\left(\mathscr{F} \otimes m^{*} \mathcal{O}_{D}\right)$ is locally free of rank $g$.

$-R^{1} q_{*}\left(\mathscr{F} \otimes m^{*} \mathcal{O}_{D}\right)=0$.

- $R^{1} q_{*}\left(\mathscr{F} \otimes m^{*} \mathcal{O}_{C}(-D)\right)$ is locally free of rank $g$. 
To prove these assertions, it is enough (using [EGA III.7]) to show that for each $s \in S, \alpha \in J C$ :

$$
\begin{aligned}
& h^{0}\left(C, \mathscr{L}_{s} \otimes L_{\alpha} \otimes \mathcal{O}_{D}\right)=g, \\
& h^{1}\left(C, \mathscr{L}_{s} \otimes L_{\alpha} \otimes \mathcal{O}_{D}\right)=0, \\
& h^{1}\left(C, \mathscr{L}_{s} \otimes L_{\alpha}(-D)\right)=g .
\end{aligned}
$$

The first and the second equality are clear; the third one follows from the choice of the $x_{i}$ and Riemann-Roch formula.

Therefore we obtain an exact sequence:

$$
E_{1} \stackrel{u}{\longrightarrow} E_{2} \longrightarrow R^{1} q_{*}(\mathscr{F}) \longrightarrow 0
$$

where $E_{1}, E_{2}$ are locally free sheaves of rank $g$ on $J C \times S$.

Let $p=(\alpha, s)$ be a point in $J C \times S$. Since the formation of $R^{1} q_{*}(\mathscr{F})$ commutes with base change, we get an exact sequence:

$$
E_{1}(p) \stackrel{u(p)}{\longrightarrow} E_{2}(p) \rightarrow H^{1}\left(C, \mathscr{L}_{s} \otimes L_{\alpha}\right) \rightarrow 0 .
$$

Since $h^{1}\left(\mathscr{L}_{s} \otimes L_{a}\right)=h^{0}\left(\mathscr{L}_{s} \otimes L_{a}\right)$ by Riemann-Roch, we conclude that $h^{0}\left(\mathscr{L}_{s} \otimes L_{\alpha}\right)$ $\geqq 1$ if and only if det $(u(p))=0$.

We define the divisor $Z$ on $J C \times S$ by the equation $\operatorname{det}(u)=0$. Then, by construction, for each $s$ in $S, Z_{s}$ is the locus of line bundles $M$ in $J C$ with $h^{0}\left(\mathscr{L}_{s} \otimes M\right)$ $\geqq 1$. Since by 2.1 (ii), for each $s$ in $S$ one has $Z_{s} \neq J C, Z$ is flat on $S$. Since:

$$
f_{*}\left(L_{1}\right) \otimes M \cong f_{*}\left(L_{1} \otimes f^{*} M\right)
$$

one has $Z_{s_{1}}=\left(f^{*}\right)^{-1}\left(\Theta^{\prime}\right)$, where $\Theta^{\prime}=\left\{M \in J N, h^{0}\left(L_{1} \otimes M\right) \geqq 1\right\}$; and also $Z_{s_{0}}=\Theta_{L}$. So $\Theta_{L}$ is algebraically equivalent to $\left(f^{*}\right)^{-1}\left(\Theta^{\prime}\right)$.

Fix an $L$ as in the Proposition, and put $\Theta_{L}=\Theta$. The divisor $\Theta$ on $J C$ defines a group homomorphism:

$$
\lambda:\left\{\begin{aligned}
J C(k) & \rightarrow \operatorname{PiC}(J C) \\
a & \mapsto \mathcal{O}_{J C}\left(\Theta_{a}-\Theta\right)
\end{aligned}\right.
$$

([L, p. 75]); here $J C(k)$ denotes the group of rational points of $J C$. Similarly the divisor $\Theta^{\prime}$ on $J N$ defines a morphism of algebraic groups:

$$
\mu: J N \rightarrow \underline{\operatorname{Pic}}^{0}(J N)=\widehat{N} .
$$

\section{Corollary. The diagram:}

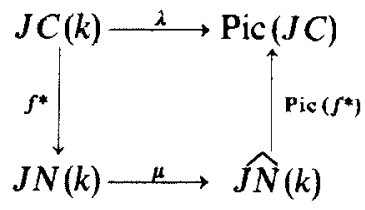

is commutative. 
The corollary is an immediate consequence of Proposition 2.2 and Proposition 4 in [L, p. 75].

(2.4) Remark. Let $C \rightarrow S$ be a flat family of curves of genus $g$, with at most ordinary double points, and let $J C \rightarrow S$ be the corresponding flat family of Jacobian varieties ( $J C$ is an algebraic space: see [A]). Locally over $S$ for the étale topology, one can find a line bundle $L$ on $C$ such that $\operatorname{deg}\left(L_{s}\right)=g-1$ and $h^{0}\left(C_{s}, L_{s}\right)=0$ for each $s$ in $S$. Then, for each $s$ in $S$, the multidegree of $L_{s}$ satisfies condition (i) of Lemma 2.1. One sees as in the proof of Proposition 2.2 that the divisors $\Theta_{L_{s}}$ on $J C_{s}$ fit together to define a divisor $\Theta$ on $J C$, flat over $S$, such that $\Theta_{\mid J C_{s}}=\Theta_{L_{s}}$ for each $s$.

\section{Generalized Prym Varieties: Definition}

Throughout the rest of this paper, $\tilde{C}$ is a connected curve with only ordinary double points, $\tilde{f}: \tilde{N} \rightarrow \tilde{C}$ its normalization, $\imath: \tilde{C} \rightarrow \tilde{C}$ an involution $\left(l^{2}=\mathrm{Id}\right)$.

(3.1) Lemma. The quotient curve $\tilde{C} /(l)$ has only ordinary double points.

Proof. We have only to check what happens at a singular point $s$ of $\tilde{C}$ fixed under the involution. Let $\mathcal{O}_{s}$ be the local ring of $\tilde{C}$ at $s$; the completion $\hat{\mathcal{O}}_{s}$ can be identified with $k[[u, v]] /(u v)$. If the involution exchanges the two branches of $\tilde{C}$ at $s$, one can choose $u, v$ so that $\imath^{*} u=v, \imath^{*} v=u$; then the subring of invariants in $\hat{\mathcal{O}}_{\mathrm{s}}$ is the ring of formal power series in $u+v$, which is regular. If the branches at $s$ are not exchanged, one can choose $u, v$ so that $l^{*} u=-u, l^{*} v=-v$; hence the subring of invariants is $k\left[\left[u^{2}, v^{2}\right]\right]$, which is isomorphic to $k[[x, y]] /(x y)$.

We now assume:

(*) The fixed points of $\iota$ are exactly the singular points, and at a singular point the two branches are not exchanged under $t$.

We note $C=\tilde{C} /(l)$ the quotient curve, $\pi: \tilde{C} \rightarrow C$ the projection, $f: N \rightarrow C$ the normalization of $C, \pi^{\prime}: \tilde{N} \rightarrow N$ the morphism deduced from $\pi$ (so that $f \circ \pi^{\prime}$ $=\pi \circ \tilde{f})$ :

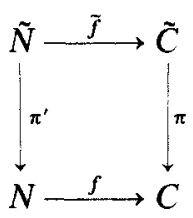

$\pi^{\prime}$ is a two-sheeted covering, ramified at the points $x_{i}, y_{i}$ of $\tilde{N}$ which lie over singular points $z_{i}$ of $\tilde{C}$.

(3.2) Lemma. $\pi^{*} \omega_{C} \cong \omega_{\tilde{C}}$.

Proof. $\omega_{\tilde{C}}$ is the sheaf of forms $\tilde{\omega}$ on $\tilde{N}$, regular except for simples poles at the $x_{i}$ and $y_{i}$, with $\operatorname{Res}_{x_{i}}(\tilde{\omega})+\operatorname{Res}_{y_{i}}(\tilde{\omega})=0$. Since $\pi$ is etale outside the singular points, and $\operatorname{Res}_{x_{i}} \pi^{*} \omega=2 \operatorname{Res}_{\pi x_{i}} \omega$ for a form $\omega \in \omega_{C}$, one gets $\operatorname{div}\left(\pi^{*} \omega\right)=\pi^{*} \operatorname{div}(\omega)$, hence $\pi^{*} \omega_{\mathcal{c}} \cong \omega_{\tilde{\mathbf{C}}}$. 
One should notice that $\pi$ is not a two-sheeted covering in the usual sense; in fact, $\pi$ is not flat at the singular points. From Lemma 3.2 we obtain: $p_{a}(\tilde{C})$ $=2 p_{a}(C)-1$.

We shall need some facts about Cartier divisors on $\tilde{C}$ and $C$. Let $\tilde{K}$ (resp. $K$ ) be the ring of rational functions on $\tilde{C}$ (resp. $C$ ), that is the product of the fields of functions of the components. The group of Cartier divisors on $\tilde{C}$ is:

$$
\operatorname{Div}(\tilde{C})=\underset{x \in \tilde{C}_{\text {reg }}}{\bigoplus} \mathbf{Z} \cdot x+\underset{s \text { singular }}{\oplus} \tilde{K}_{s}^{*} / \mathcal{O}_{s}^{*}
$$

Let $s$ be a singular point of $\tilde{C}, s_{1}, s_{2}$ the two points of $\tilde{N}$ lying over $s, v_{1}, v_{2}$ the corresponding valuations of $\tilde{K}$. One has an exact sequence:

$$
0 \rightarrow k^{*} \rightarrow \tilde{K}_{s}^{*} / \mathcal{O}_{s}^{*} \stackrel{v_{1}, v_{2}}{\longrightarrow} \mathbf{Z} \oplus \mathbf{Z} \rightarrow 0
$$

(the kernel is identified with $k^{*}$ by $f \mapsto \frac{f\left(s_{1}\right)}{f\left(s_{2}\right)}$ ).

It is convenient to split this exact sequence by choosing uniformizing parameters $t_{1}$ and $t_{2}$ at $s_{1}$ and $s_{2}$, thus getting an isomorphism: $\tilde{K}_{s}^{*} / \mathcal{O}_{s}^{*} \stackrel{\sim}{\longrightarrow} k^{*} \times \mathbf{Z} \times \mathbf{Z}$. With this identification, assuming $\imath^{*} t_{1}=-t_{1}, \imath^{*} t_{2}=-t_{2}$, the action of $t$ on $\tilde{K}_{s}^{*} / \mathcal{O}_{s}^{*}$ is simply:

$$
l^{*}(z, m, n)_{s}=\left((-1)^{m+n} z, m, n\right)_{s} .
$$

The norm of $\tilde{K} / K$ maps $\mathcal{O}_{\tilde{C}}$ into $\mathcal{O}_{C}$, thus gives a diagram of exact sequences:

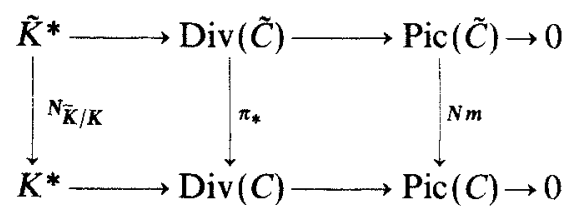

where:

- $\pi_{*}$ is the direct image under $\pi: \pi_{*}\left(\sum_{i} x_{i}\right)=\sum_{i} \pi x_{i}$ for $x_{i} \in C_{\text {reg }}$,

$\pi_{*}\left((z, m, n)_{s}\right)=\left((-1)^{m+n} z^{2}, m, n\right)_{\pi s}$ (follows from the formula for $\left.\imath^{*}\right)$.

- Nm: $\operatorname{Pic}(\tilde{C}) \rightarrow \operatorname{Pic}(C)$ is the usual norm for line bundles ([EGA II.6.5]), which induces a morphism of algebraic groups $N m: J \tilde{C} \rightarrow J C$.

(3.3) Lemma. If $L$ is a line bundle on $\tilde{C}$ such that $N m L \cong \mathcal{O}_{C}$, then $L \cong M \otimes \imath^{*} M^{-1}$ for some line bundle $M$ on $\tilde{C}$. Moreover $M$ can be chosen of multidegree $(0, \ldots, 0)$ or $(1,0, \ldots, 0)$.

Proof. As in [M 1], Lemma 1, we get $L=\mathcal{O}(D)$ where $\pi_{*}(D)=0$. Writing $D=\sum_{i} x_{i}$ $+\sum_{s \text { singular }}\left(z_{s}, m_{s}, n_{s}\right)$, we get that $D$ is a linear combination of divisors $x-i x$, for $x \in \tilde{C}_{\mathrm{reg}}$, and $(-1,0,0)_{s}$ at singular points $s$; but $(-1,0,0)=(1,0,1)-\imath^{*}(1,0,1)$, hence $D=E-\imath^{*} E$ for some divisor $E$ and $L \cong M \otimes \imath^{*} M^{-1}$ with $M=\mathcal{O}(E)$. We may replace $M$ by $M \otimes \pi^{*} N$ for any line bundle $N$ on $C$, hence assume $\operatorname{deg}(M)$ $=\left(\varepsilon_{1}, \ldots, \varepsilon_{c}\right)$ where $\varepsilon_{i}=0$ or 1 . Since $(\operatorname{Id}-\imath)^{*}(1,1,-1)=0$ and $\tilde{C}$ is connected, we can further normalize $M$ as in the statement of the lemma. 
We denote by $P$ (resp. $P_{1}$ ) the variety of line bundles in $\operatorname{Ker}(\mathrm{Nm})$ of the form $M \otimes \iota^{*} M^{-1}$ with $\operatorname{deg}(M)=(0, \ldots, 0)$ (resp. $\left.(1,0, \ldots, 0)\right)$. Note that $P$ is a connected algebraic subgroup of $J \tilde{C}$.

(3.4) Proposition. Fix a line bundle $L_{0}$ on $C$ with $L_{0}^{2} \cong \omega_{C}$. The function:

$$
L \mapsto h^{0}\left(L \otimes \pi^{*} L_{0}\right)
$$

is constant $\bmod 2$ on $P$ and on $P_{1}$, and takes opposite parity on $P$ and $P_{1}$.

Notice that such an $L_{0}$ always exists, since the hypothesis (*) insures that $\operatorname{deg} \omega_{\mathcal{C}_{\mid C_{i}}}$ is even for all $i$.

Proof. We first prove that $h^{0}\left(L \otimes \pi^{*} L_{0}\right)=h^{0}\left(L_{0} \otimes \pi_{*} L\right)$ is constant $\bmod 2$ when $L$ varies in a connected algebraic family; for this we exhibit a non-degenerate quadratic form $S^{2}\left(L_{0} \otimes \pi_{*} L\right) \rightarrow \omega_{C}$ (or, what amounts to the same, $S^{2} \pi_{*} L \rightarrow O_{C}$ ) and apply Theorem 1.1. The norm induces a quadratic form:

$$
S^{2} \pi_{*} L \rightarrow N m(L) \cong \mathcal{O}_{C}
$$

which can be identified locally with:

$$
Q:\left\{\begin{aligned}
S^{2} \pi_{*} \mathcal{O}_{\tilde{c}} & \rightarrow \mathcal{O}_{\mathrm{C}} \\
h & \mapsto N m(h)
\end{aligned}\right.
$$

and $Q$ is easily seen to be non-degenerate by local computation. The proof in [M 1] that $h^{0}\left(L \otimes \pi^{*} L_{0}\right)$ takes opposite parity on $P$ and $P_{1}$ applies in a straightforward manner to our case (use Lemma 3.2 in Step II).

(3.5) Proposition. $P$ is an abelian variety of dimension $p_{a}(C)-1$.

Proof. We look at the diagram:

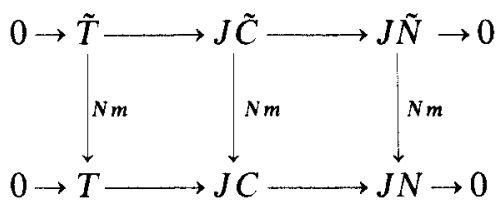

where $\tilde{T}$ and $T$ are the groups of classes of divisor of multidegree $(0, \ldots, 0)$ with singular support; $\pi^{*}$ induces an isomorphism of $T$ onto $\tilde{T}$, hence, since $N m \circ \pi^{*}=2$, $N m_{\mid \tilde{T}}$ is surjective and $\operatorname{Ker} N m_{\mid \tilde{T}}=\tilde{T}_{2}=$ points of order 2 in $\tilde{T}$ \}.

Thus one gets an exact sequence:

$$
0 \rightarrow \tilde{T}_{2} \rightarrow P \times \mathbf{Z} / 2 \stackrel{\mathrm{g}}{\rightarrow} R \rightarrow 0
$$

where $R=\operatorname{Ker} N m_{\mid J \tilde{N}}$ is a complete subvariety of $J \tilde{N}$; therefore $P$ is complete, reduced and connected, hence an abelian variety.

(3.6) Remark. $R$ is an abelian variety, called in [M2] the Prym variety associated to the ramified two-sheeted covering $\tilde{N} \rightarrow N$. Notice that $g: P \rightarrow R$ is an isogeny, but not an isomorphism if the dimension $t$ of $T$ is greater than 2: in fact \# $\operatorname{ker}(\mathrm{g})$ $=2^{t-1}$. 
Using the line bundle $\pi^{*} L_{0}$ we define a divisor $\Theta$ on $J \tilde{C}(\S 2)$.

(3.7) Theorem. $\Theta$ induces twice a principal polarization on $P$.

The statement of the theorem means that $\Theta^{\prime}=\Theta_{\mid P}$ is algebraically equivalent to $2 \Xi$, where $\Xi$ is an ample divisor with $h^{0}(\mathcal{O}(\Xi))=1$; or, equivalently, that the morphism

$$
\rho:\left\{\begin{array}{l}
P \rightarrow \hat{P} \\
a \mapsto \mathcal{O}_{P}\left(\Theta_{a}^{\prime}-\Theta^{\prime}\right)
\end{array}\right.
$$

is twice an isomorphism. By Corollary 2.3 , we have a commutative diagram:

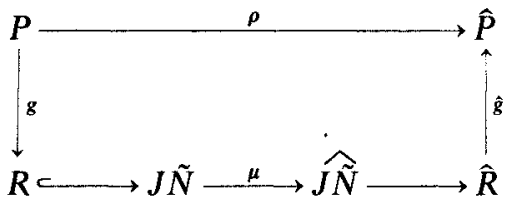

We first need the following lemma:

(3.8) Lemma. Let $h: A \rightarrow B$ be an isogeny of abelian varieties, $\beta: B \rightarrow \widehat{B}$ a principal polarization on $B, \alpha=\hat{h} \circ \beta \circ h$ the pullback of $\beta$ on $A$.

Assume:

(i) $\operatorname{Ker} h \subset A_{2}$, the set of points of order two in $A$;

(ii) $h\left(A_{2}\right)$ is totally isotropic maximal with respect to the symmetric pairing defined by $\beta$ on $B_{2}$.

Then $\operatorname{Ker} \alpha=A_{2}$.

Proof of the Lemma. Let us consider $A_{2}$ and $B_{2}$ as vector spaces over $F_{2}$, and denote by $A_{2}^{*}$ and $B_{2}^{*}$ the dual spaces. Cartier duality provides us with a commutative diagram:

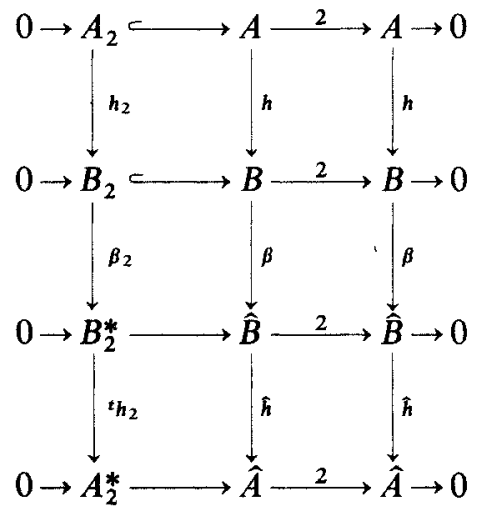

where the horizontal rows are exact.

It follows from (i) that $\operatorname{Ker} \hat{h} \subset(\hat{B})_{2}$, hence: $\beta^{-1}(\operatorname{Ker} \hat{h})=\beta_{2}^{-1}\left(\operatorname{Ker}^{t} h_{2}\right)=$ $\beta_{2}^{-1}\left(\left(\operatorname{Im} h_{2}\right)^{*}\right)=\left(\operatorname{Im} h_{2}\right)^{\perp}$, where the sign $\perp$ means orthogonality with respect to the pairing on $B_{2}$ defined by $\beta$. 
Now, (ii) implies that $\left(\operatorname{Im} h_{2}\right)^{\perp}=\operatorname{Im} h_{2}$, so that: $\operatorname{Ker} \alpha=h^{-1}\left(\operatorname{Im} h_{2}\right)=A_{2}+\operatorname{Ker} h$ $=A_{2}$.

Proof of Theorem (3.7). We apply the lemma to the isogeny $h: P \times J N \rightarrow J \tilde{N}$ and the principal polarization on $J \tilde{N}$. We check the conditions (i) and (ii):

(i) An element of $\operatorname{Ker}(h)$ is a pair $(L, M)$ with $L \in P, M \in J N$ and $\tilde{f}^{*} L \otimes \pi^{* *} M$ $\cong \mathcal{O}_{\tilde{N}}$. Write $M=f^{*} M^{\prime}$ for some $M^{\prime} \in J C$; then $L \otimes \pi^{*} M^{\prime} \in \operatorname{ker} f^{*}=\tilde{T}$, hence since $\tilde{T}=\pi^{*}(T), L \cong \pi^{*} M^{\prime \prime}$ for some $M^{\prime \prime} \in J C$. Using $N m(L) \cong \mathcal{O}_{C}$ one gets $M^{\prime \prime 2}=\mathcal{O}_{C}$, hence $L^{2}=\mathcal{O}_{\tilde{C}}$ and $M^{2}=\mathcal{O}_{N}$.

(ii) Let $e_{2, \bar{N}}$ (resp. $e_{2, N}$ ) the pairing defined on $J \tilde{N}_{2}$ (resp. $J N_{2}$ ) by the polarization. $N m$ and $\pi^{* *}$ are dual with respect to the polarizations on $J \tilde{N}$ and $J N$, therefore:

$$
e_{2, \tilde{N}}\left(\pi^{\prime *} a, b\right)=e_{2, N}(a, N m(b)) \quad \text { for } a \in J N_{2}, b \in J \tilde{N}_{2} .
$$

In particular, $\pi^{*} J N_{2}$ is orthogonal to both itself and $\tilde{f}^{*} P_{2}$. Now if $L, M \in P_{2}$, Corollary 1.2 gives:

$$
\begin{aligned}
\ln e_{2, \bar{N}}\left(\tilde{f}^{*} L, \tilde{f}^{*} M\right)= & h^{0}\left(\pi^{*} L_{0}\right)+h^{0}\left(\pi^{*} L_{0} \otimes L\right)+h^{0}\left(\pi^{*} L_{0} \otimes M\right) \\
& +h^{0}\left(\pi^{*} L_{0} \otimes L \otimes M\right)(\bmod 2) \\
\equiv & 4 h^{0}\left(\pi^{*} L_{0}\right) \bmod 2 \text { by Proposition 3.6, }
\end{aligned}
$$

hence the subspace $h\left(P_{2} \times J N_{2}\right)$ is totally isotropic in $J \tilde{N}_{2}$. To prove that it is maximal, we have only to check that:

$$
\operatorname{dim}_{\mathbf{F}_{2}}\left(h\left(P_{2} \times J N_{2}\right)\right)=\frac{1}{2} \operatorname{dim}_{\mathbf{F}_{2}}\left(J \tilde{N}_{2}\right) .
$$

Since $\operatorname{dim}_{\mathbf{F}_{2}}\left(P_{2} \times J N_{2}\right)=\operatorname{dim}_{\mathbf{F}_{2}}\left(J \tilde{N}_{2}\right)$, it is equivalent to show that:

$$
\operatorname{dim}_{\mathbf{F}_{2}}(\operatorname{Ker} h)=\frac{1}{2} \operatorname{dim}_{\mathbf{F}_{2}}\left(J \tilde{N}_{2}\right) \text {. }
$$

We have shown in the proof of (i) that:

$\operatorname{Ker} h=\left\{\left(\pi^{*} a, f^{*} a\right)\right.$ where $a \in J C_{2}$ is such that $\left.\pi^{*} a \in P\right\}$.

Since $\pi^{*}$ (resp. $f^{*}$ ) is injective when $C$ is singular (resp. non-singular), we conclude that Ker $h$ is isomorphic to the group of points $a \in J C_{2}$ such that $\pi^{*} a \in P$; this group is the kernel of the linear form given by the composition:

$$
\varphi: J C_{2} \stackrel{\pi^{*}}{\longrightarrow} \operatorname{Ker}(\mathrm{Nm}) \longrightarrow \operatorname{Ker}(\mathrm{Nm}) / P \stackrel{\sim}{\longrightarrow} \mathbf{F}_{2}
$$

(where the last isomorphism is given by Lemma 3.3).

We now prove that $\varphi \neq 0$. If $C$ has a singular point $s$, let $D_{s}$ be the divisor $(-1,0,0)_{s}$ and $d$ the class of $\mathcal{O}_{C}(D)$ in $J C$; then $d \in J C_{2}$ and since $\pi^{*} D_{s}=(-1,0,0)_{s}$, one has $\pi^{*} d \in P_{1}$, hence $\varphi(d)=1$. If $C$ is non-singular, it follows from [M2] (or directly from Corollary 1.2$)$ that:

$$
\varphi(a)=(\varepsilon \cdot a) \quad \text { for each } a \in J C_{2},
$$

where $\varepsilon$ is the only non-zero element of $\operatorname{Ker}\left(\pi^{*}\right)$; in particular $\varphi \neq 0$. Therefore, in any case, if we put $t=\operatorname{dim}(T)=\operatorname{dim}(\tilde{T})$, we obtain:

$$
\operatorname{dim}_{\mathbf{F}_{2}}(\operatorname{Ker} h)=\operatorname{dim}_{\mathbf{F}_{2}}\left(J C_{2}\right)-1=2 g-t-1
$$

while $\operatorname{dim}_{\mathbf{F}_{2}}\left(J \tilde{N}_{2}\right)=2 \operatorname{dim}(J \tilde{N})=2(2 g-1-t)$, which achieves the proof of (ii). 
Therefore the lemma applies and gives:

$\operatorname{Ker}(\hat{h} \circ \mu \circ h)=P_{2} \times J N_{2}$.

But the polarization $\alpha=\hat{h} \circ \mu \circ h$ can be written as a sum of four morphisms:

$$
\rho: P \rightarrow \widehat{P} ; \quad \sigma: P \rightarrow \widehat{J N} ; \quad \tau: J N \rightarrow \hat{P} ; \quad v: J N \rightarrow \widehat{J N} .
$$

Since $\pi^{*}$ and $N m$ are dual to each other with respect to the principal polarizations of $J \tilde{N}$ and $J N$ ([M2]), we find that $\sigma=0$; then from $\hat{\alpha}=\alpha$ we deduce that $\tau=\hat{\sigma}=0$. We conclude that $\operatorname{Ker}(\rho)=P_{2}$, which proves the proposition.

(3.9) Definition. The abelian variety $P$, together with the principal polarization $\Xi$ defined by $2 \Xi \equiv \Theta_{\mid P}$ in $N S(P)$, is the (generalized) Prym variety associated to $(\tilde{C}, \imath)$.

Actually, as in the non-singular case, the relation between $\Theta$ and $\Xi$ can be made much more precise:

(3.10) Proposition. Choose $L_{0}$ such that $h^{0}\left(\pi^{*} L_{0}\right)$ is even (and $L_{0}^{2} \cong \omega_{C}$ ). Then, with the preceding notation: $\Theta_{\mid P}=2 \Xi$, where $\Xi \subset P$ is a divisor in the class of the principal polarization on $P$.

Notice that one can always find such an $L_{0}$ : if $s$ is a singular point of $C, D$ the divisor $(-1,0,0)_{s}$, then $h^{0}\left(\pi^{*}\left(L_{0}(D)\right)\right) \equiv h^{0}\left(\pi^{*} L_{0}\right)+1(\bmod 2)$, by Lemma 3.3 and Proposition 3.4.

To prove the proposition, we need Riemann's singularity theorem for singular curves. We state a more general result:

(3.11) Proposition. Let $C$ be a curve, $J C^{*}$ the variety of line bundles $L$ on $C$ such that $2 \operatorname{deg} L=\operatorname{deg} \omega_{C}, \Theta$ the divisor of line bundles $M$ in $J C^{*}$ such that $h^{0}(M) \geqq 1$, $L \in \Theta, \varphi$ the pairing:

$$
H^{0}(L) \otimes H^{0}\left(\omega_{C} \otimes L^{-1}\right) \rightarrow H^{0}\left(\omega_{C}\right) .
$$

Choose a basis $\left(s_{i}\right)$ of $H^{0}(L)$, a basis $\left(t_{j}\right)$ of $H^{0}\left(\omega_{C} \otimes L^{-1}\right)$; identify $H^{0}\left(\omega_{c}\right)$ to the dual of the tangent space $T$ to $J C^{*}$ at $L$. Assume the function $\operatorname{det}\left(\varphi\left(s_{i} \otimes t_{j}\right)\right)$ is not identically zero on $T$; then it defines a hypersurface in $T$ which is equal to the tangent cone to $\Theta$ at $L$. In particular the multiplicity of $\Theta$ at $L$ is $h^{0}(L)$.

Proof of Proposition (3.11). The proposition is proved in Kempf's thesis (see [Sz]) when $C$ is irreducible; the argument can be adapted to the general case as follows: let $\mathscr{P}$ be a Poincaré bundle on $C \times J C^{*}, q: C \times J C^{*} \rightarrow J C^{*}$ the projection. Put $h^{0}(L)=m$; choosing $m$ non-singular points $x_{1}, \ldots, x_{m}$ such that $H^{0}\left(C, L\left(-\sum x_{i}\right)\right)$ $=(0)$, we get as in Proposition 2.1 an exact sequence in a neighborhood $U$ of $L$ in $J C^{*}$ :

$$
\mathcal{O}_{U}^{m} \stackrel{u}{\longrightarrow} \mathcal{O}_{U}^{m} \longrightarrow R^{1} q_{*}(\mathscr{P}) \rightarrow 0
$$

and a local equation for $\Theta$ is $\operatorname{det}(u)=0$.

Since $h^{\circ}(L)=m$, the coefficients $u_{i j}$ of $u$ are zero at $L$; the tangent cone to $\Theta$ at $L$ is given by the determinant of the first-order terms of the $u_{i j}$-unless this determinant is identically zero. In other words, let $t$ be a tangent vector to $J C^{*}$ 
at $L ; t$ corresponds to a morphism Spec $k[\varepsilon] \rightarrow J C^{*}\left(\varepsilon^{2}=0\right)$, or equivalently to a line bundle $L_{\varepsilon}$ on $C_{\varepsilon}=C \times \operatorname{Spec} k[\varepsilon]$; and $t$ is tangent to $\Theta$ at $L$ if $\operatorname{dim}_{k} \operatorname{Ker}\left(t^{*} u\right)$ $\geqq m+1$. Now by construction, $\operatorname{Ker}\left(t^{*} u\right) \cong H^{0}\left(C_{\varepsilon}, L_{\varepsilon}\right)$; the tangent vector $t$, viewed as an element of $H^{1}\left(C, \mathcal{O}_{C}\right) \cong \operatorname{Ext}_{\mathscr{O}_{C}}^{1}(L, L)$, corresponds to an extension of sheaves:

$$
0 \rightarrow L \rightarrow L_{\varepsilon} \rightarrow L \rightarrow 0
$$

which gives:

$$
0 \rightarrow H^{0}(L) \rightarrow H^{0}\left(L_{\varepsilon}\right) \rightarrow H^{0}(L) \rightarrow H^{1}(L) .
$$

Thus $t$ belongs to the tangent cone if and only if the map $H^{0}(L) \rightarrow H^{1}(L)$ defined by cup-product with $t$ is not an isomorphism. By choosing a basis for $H^{\circ}(L)$ and $H^{0}\left(\omega_{C} \otimes L^{-1}\right)$ one finds the statement of the proposition.

Proof of Proposition (3.10). We must check that for line bundles $L$ in $J \tilde{C}^{*}$ satisfying $\imath^{*} L \cong \omega_{\bar{c}} \otimes L^{-1}$, the determinant given in Proposition 3.11 is not identically zero. But the vanishing of $\operatorname{det}\left(\varphi\left(s_{i} \otimes t_{j}\right)\right)$ implies that there is a $s \in H^{0}(\tilde{C}, L)$ such that $\varphi(s \otimes t)=0$ for all $t \in H^{0}\left(\tilde{C}, \omega_{\tilde{c}} \otimes L^{-1}\right)$; since $\varphi\left(s \otimes l^{*} s\right)$ is non-zero, this is impossible. Thus we can use Riemann's singularity theorem, and the argument in [M2, p. 342] applies identically.

(3.12) Remark. To avoid the choice of a theta-characteristic $L_{0}$ as in Proposition 3.10 , it is often convenient to look at the Prym variety in $J C^{*}$, after translation by $\pi^{*} L_{0}$ : thus the Prym variety becomes the variety of line bundles $L$ in $J C^{*}$ such that $N m(L) \cong \omega_{\mathrm{C}}$ and $h^{0}(L)$ is even, $\Xi$ is the divisor of effective line bundles in $P$, and $\Theta_{1 P}=2 \Xi$.

\section{Dimension of Sing $\Xi$}

Keeping the notation of $\S 3$, we denote by $\tilde{C}$ a connected curve of genus $2 g-1$ with ordinary double points, $l$ an involution of $\tilde{C}$ satisfying condition $(*), C$ the quotient curve (of genus $g$ ). Our aim is to extend Mumford's description of Sing $\Xi$ ([M2]) to the Prym variety and its polarization defined in $\S 3$. According to Remark 3.12, we look at the situation in $J \tilde{C}^{*}$; we denote:

$$
\begin{aligned}
& P=\left\{\text { line bundles } L \text { on } \tilde{C}, N m(L) \cong \omega_{C}, h^{0}(L) \text { even }\right\}, \\
& \Xi=\left\{L \text { in } P, h^{0}(L) \geqq 2\right\} .
\end{aligned}
$$

(4.1) Lemma. $A$ line bundle $L$ in $P$ belongs to Sing $\Xi$ if and only if :

(i) either $h^{0}(L) \geqq 4$,

(ii) or $h^{0}(L)=2$, and for a basis $\{s, t\}$ of $H^{0}(\tilde{C}, L)$ one has:

$$
\imath^{*} s \otimes t=s \otimes \imath^{*} t \quad \text { in } \quad H^{0}\left(\tilde{C}, L \otimes \imath^{*} L\right)=H^{0}\left(\tilde{C}, \omega_{\tilde{C}}\right) .
$$

Proof. By Proposition 3.10, a point $L$ in $P$ belongs to $\operatorname{Sing} \Xi$ either if it is of multiplicity $\geqq 3$, or if it is of multiplicity 2 and the tangent space to $P$ is contained in 
the tangent cone to $\Theta$ at $L$. The first case gives (i); in the second case, we can apply the analysis in [M2, p. 343], using Proposition 3.11, and find condition (ii).

We first get rid of case (i) following [M2].

(4.2) Proposition. Let $Z$ be an irreducible component of $\operatorname{Sing} \Xi$ with $\operatorname{dim} Z \geqq g-5$. Then, a generic line bundle $L$ in $Z$ has the property:

(P) There exist two linearly independent sections $s, t$ in $H^{0}(\tilde{C}, L)$ such that $\imath^{*} s \otimes t=s \otimes \imath^{*} t$.

Proof. The proof in [M 2, p. 345] applies identically once one knows the following lemma:

(4.3) Lemma. Let $X$ be a curve of genus $g$. Let us denote by $G_{d}^{r}$ the variety of line bundles $L$ on $X$ with $\operatorname{deg} L=d, h^{0}(L) \geqq r+1$. Let $Z$ be an irreducible subvariety of $G_{d}^{r}$, L a line bundle in $Z$ with $h^{0}(L)=r+1, \varphi_{L}$ the pairing:

$$
\varphi_{L}: H^{0}(L) \otimes H^{0}\left(\omega_{X} \otimes L^{-1}\right) \rightarrow H^{0}\left(\omega_{X}\right) .
$$

Then

$\operatorname{dim} Z \leqq g-\operatorname{dim} \operatorname{Im} \varphi_{L}$.

Proof as in [S-D, p. 162].

We now begin the study of line bundles with property $(\mathrm{P})$. We fix some notation. If $L$ is a line bundle on a curve $X$, we denote by $|L|$ the set of effective divisors $D$ such that $\mathcal{O}(D) \cong L$; this is an open set in $\mathbf{P}\left(H^{0}(X, L)\right.$ ) (which may be different from $\mathbf{P}\left(H^{0}(X, L)\right)$ if $X$ is reducible). We shall say for convenience that $L$ is nonsingular if $|L|$ contains a divisor with non-singular support (or equivalently, if at each double point $x$ of $X$, there is a global section $s$ of $L$ such that $s(x) \neq 0)$.

(4.4) Lemma. Let $L$ be a line bundle on $\tilde{C}$ with property (P); assume that at each double point of $C$, either $s$ or $t$ do not vanish. Then $L \cong \pi^{*} M(E)$, where:

- $M$ is a non-singular line bundle on $C$ with $h^{0}(M) \geqq 2$.

- $E$ is a divisor on $\tilde{C}$ with non-singular support.

- $\pi_{*} E \in\left|\omega_{c} \otimes M^{-2}\right|$; in particular $\omega_{C} \otimes M^{-1}$ and $\omega_{c} \otimes M^{-2}$ are non-singular line bundles.

Proof. One can suppose that $s$ and $t$ are both non-zero at each double point of $\tilde{C}$. Put $\varphi=s / t$; since $\imath^{*} \varphi=\varphi$, one gets $\varphi=\pi^{*} \psi$, where $\psi$ is a rational function on $C$. Let $E$ be the divisor of common zeros of $s$ and $t, Z(s)$ (resp. $Z(\varphi), Z(\psi)$ ) the divisor of zeros of $s$ (resp. $\varphi, \psi$ ); all these divisors have non-singular support and one has:

$$
Z(s)=Z(\varphi)+E \text { with } Z(\varphi)=\pi^{*} Z(\psi) .
$$

This gives $L \cong \pi^{*} M(E)$, with $M=\mathcal{O}_{C}(Z(\psi))$; the last statement follows from the isomorphism $N m(L) \cong \omega_{C}$.

(4.5) Notice that the argument is still valid if the involution $\imath$ has some nonsingular fixed points.

We are thus led to study the dimension of the locus of line bundles $M$ on a curve $C$ with ordinary double points, such that $h^{0}(M) \geqq 2$ and $M, \omega_{C} \otimes M^{-1}$ and $\omega_{c} \otimes M^{-2}$ are non-singular. We need some preliminary lemmas: 
(4.6) Lemma. Let $L, M$ be two non-singular line bundles on $C, \varphi$ the pairing: $H^{0}(L) \otimes H^{0}(M) \rightarrow H^{0}(L \otimes M)$.

Then $\operatorname{dim} \operatorname{Im} \varphi \geqq h^{0}(L)+h^{0}(M)-1$.

Proof. The lemma follows from the fact that $|L|$ and $|M|$ are non-empty, and the morphism:

$$
|L| \times|M| \rightarrow|L \otimes M|
$$

is generically finite.

(4.7) Lemma. Let $L$ be a non-singular line bundle on $C$; suppose $\omega_{C} \otimes L^{-1}$ is nonsingular. Then:

$$
h^{\circ}(L) \leqq \frac{\operatorname{deg} L}{2}+1
$$

If equality holds, then either $L=\mathcal{O}_{C}$ or $L=\omega_{C}$ or there exists on $C$ a non-singular line bundle $M$ with $\operatorname{deg} M=h^{0}(M)=2$ (we'll say for short that " $C$ has a non-singular $g_{2}^{1}$ ”).

Proof. The first statement follows from Lemma 4.6 and the Riemann-Roch theorem; the second statement is proved as in [S-D, p. 159], noting that if one chooses $D^{\prime} \in \mid \omega_{C} \otimes L^{-1} /$ with non-singular support, then $\mathcal{O}_{C}\left(\left(D, D^{\prime}\right)\right)$ and $\omega_{C}\left(-\left(D, D^{\prime}\right)\right)$ are non-singular.

(4.8) Lemma. Let $Z$ be an irreducible subvariety of $G_{d}^{r},(0<d<2 g-2)$ such that for $L$ generic in $Z$ both $L$ and $\omega_{C} \otimes L^{-1}$ are non-singular. Then $\operatorname{dim}(Z) \leqq d-2 r$. Moreover if $\omega_{C} \otimes L^{-2}$ is non-singular, equality holds only if $C$ has a non-singular $\mathrm{g}_{2}^{1}$.

Proof. The first part follows from Lemmas 4.3 and 4.6. If $\operatorname{dim} Z=d-2 r$, a generic line bundle $L$ in $Z$ is generated by its global sections, and $h^{0}(L)=2$. From the exact sequence:

$$
0 \rightarrow \omega_{C} \otimes L^{-2} \stackrel{(t,-s)}{\longrightarrow}\left(\omega_{C} \otimes L^{-1}\right)^{2} \stackrel{(s, t)}{\longrightarrow} \omega_{C} \rightarrow 0
$$

we get:

$$
\operatorname{dim} \operatorname{Im} \varphi_{L}=2 h^{0}\left(\omega_{C} \otimes L^{-1}\right)-h^{0}\left(\omega_{C} \otimes L^{-2}\right),
$$

hence by Lemma 4.3:

$$
d-2 r \leqq g-2(g+r-d)+h^{0}\left(\omega_{C} \otimes L^{-2}\right)
$$

or

$$
h^{0}\left(\omega_{C} \otimes L^{-2}\right) \geqq g-d=\frac{1}{2} \operatorname{deg}\left(\omega_{C} \otimes L^{-2}\right)+1 .
$$

Since $L^{2}$ is clearly non-singular, $C$ has a non-singular $g_{2}^{1}$ by Lemma 4.7.

(4.9) Lemma. Let $C$ be a curve of genus $g$ with ordinary double points, such that for each component $C_{i}$ of $C$, the intersection number of $C_{i}$ with the rest of $C$ is even. Suppose that $C$ has no non-singular $g_{2}^{1}$. Let $Z$ be an irreducible subvariety 
of $G_{d}^{1}, 0<d \leqq g-2$; assume that for L generic in $Z, L, \omega_{c} \otimes L^{-1}$ and $\omega_{c} \otimes L^{-2}$ are non singular. Then, $\operatorname{dim} Z \leqq d-3$.

If $\operatorname{dim} Z=d-3, C$ is one of the following:

a) $C$ is trigonal (=3-sheeted covering of $\mathbf{P}^{\mathbf{1}}$ );

b) $C$ is a two-sheeted covering of a curve of genus one, and $g \geqq 6$;

c) $C$ is a plane quintic;

d) $C$ is the union of two curves $C_{1}$ and $C_{2}$, with one of the following configurations:

$\# C_{1} \cap C_{2}=2$.

\# $C_{1} \cap C_{2}=4$, and neither $C_{1}$ nor $C_{2}$ is a rational curve.

$\# C_{1} \cap C_{2}=4, C_{1}$ is rational, $C_{2}$ has a non-singular $g_{2}^{1}$ and $p_{a}\left(C_{2}\right) \geqq 4$.

$\# C_{1} \cap C_{2}=4, C_{1}$ is rational and $\omega_{C_{2}} \cong \mathcal{O}_{C_{2}}\left(\sum u_{i}\right)$, where $C_{1} \cap C_{2}=\left\{u_{1}, \ldots, u_{4}\right\}$.

(4.9.1) Proof. The first statement is contained in Lemma 4.8. Assume $\operatorname{dim} Z=d-3$, and take the smallest $d$ for which this happens (so that a generic $L$ in $Z$ is generated by its global sections). If $d=3$, we get a non-singular line bundle $L$ with $h^{0}(L)=2$, $\operatorname{deg} L=3$. $L$ defines a morphism $h: C \rightarrow \mathbf{P}^{1}$. If some union of components $C_{1}$ goes to a point under $h$, the intersection of $C_{1}$ with the rest of $C$ consists of at most 2 points (since this number is even by hypothesis, and $h$ is of degree 3 ), so we are in case d); if not, $h$ is a 3 -sheeted covering of $\mathbf{P}^{1}$ : that is case a).

(4.9.2) Assume $d \geqq 4$ (hence $g \geqq 6$ ). The exact sequence (4.8.1) gives $h^{0}\left(L^{2}\right)=d$, so that we get a $(d-3)$-dimensional subvariety of $G_{2 d}^{d-1}$. By Lemma 4.8 , this is possible only if:

(i) $d=4, \operatorname{dim} Z=1$,

(ii) $d=5, \operatorname{dim} Z=2, g \geqq 7$.

Exclusing case d), we may assume that for any decomposition $C=C_{1} \cup C_{2}$ with $\# C_{1} \cap C_{2}=n$, the following holds:

(A) $n \geqq 4$, and $n \geqq 6$ except if $C_{1}$ or $C_{2}$ is a rational curve. Moreover since $\omega_{C} \otimes L^{-2}$ must be non-singular we get:

(B) $\operatorname{deg} L_{i \mathrm{C}_{i}} \leqq p_{a}\left(C_{i}\right)-1+\frac{n}{2} \quad(i=1,2)$.

Furthermore we claim that one must have $\operatorname{deg} L_{\mid C_{i}}>0$ for any component $C_{i}$ of $C$. Namely for $L$ generic in $Z$, let us denote by $h_{L}: C \rightarrow \mathbf{P}^{1}$ the morphism defined by $L$. Let $C_{0}$ be the union of the components $C_{j}$ of $C$ such that $h_{L}\left(C_{j}\right)=\mathbf{P}^{1}$ (for $L$ generic in $Z$ ). If $h_{L}^{-1}(\{z\})$ is one-dimensional for some $z \in \mathbf{P}^{1}$ and $L$ generic, one must have $h_{L}^{-1}(\{z\}) \cap C_{0}=\left\{u_{1} \ldots u_{4}\right\}$ by $(\mathrm{A})$, and $h^{0}\left(C_{0}, L_{\mid C_{0}}\left(-\sum u_{i}\right)\right) \geqq 1$ for $L$ generic in $Z$.

Let us put $L_{\mid} C_{0}=L_{0}$. If $\operatorname{deg}(L)=4$ (case (i)), we get:

$L_{0} \cong \mathcal{O}_{C_{0}}\left(\sum u_{i}\right) \quad$ for $L$ generic in $Z$.

In order to get $\operatorname{dim} Z \geqq 1$, we must have $h^{\circ}\left(L_{0}\right) \geqq 3$.

Note that $L_{0}$ and $\omega_{C_{0}} \otimes L_{0}^{-1}$ are non-singular, and $p_{a}\left(C_{0}\right) \geqq 3$ by (B). Hence Lemma 4.7 gives:

- either $p_{a}\left(C_{0}\right)=3$ and $\omega_{C_{0}} \cong \mathscr{O}_{C_{0}}\left(\sum u_{i}\right)$

- or $p_{a}\left(C_{0}\right) \geqq 4$ and $C_{0}$ has a non-singular $g_{2}^{1}$. 
Both cases are excluded (by d)). If $\operatorname{deg}(L)=5$, we find:

$$
L_{0} \cong \mathcal{O}_{c_{0}}\left(\sum u_{i}+x\right) ; \quad x \in C_{0} .
$$

Since $\operatorname{dim} Z \geqq 2, x$ must be a generic point of some component of $C_{0}$; but this contradicts the fact that $L$ is generated by its global sections.

We conclude finally that $C_{0}=C$, i.e. $\operatorname{deg} L_{\mid C_{i}}>0$ for any component $C_{i}$.

(4.9.3) Now if possibility i) holds, we apply the argument in [M2, p. 349]; note that the line bundle $M$ can be chosen non-singular. So we get a morphism $h: C \rightarrow \mathbf{P}^{2}$ such that for a generic $L$ in $Z$, the morphism $C \rightarrow \mathbf{P}^{1}$ defined by $L$ is the composition of $h$ with a projection from $\mathbf{P}^{2}$ to $\mathbf{P}^{1}$. We denote by $\left(C_{i}^{\prime}\right)$ the irreducible components of $C^{\prime}=h(C)$,

$$
d_{i}=\operatorname{deg} C_{i}^{\prime}, \quad r_{i}=\operatorname{deg} h_{\left(h^{-1}\left(C_{i}^{i}\right)\right.} .
$$

One has:

$$
\operatorname{deg} L_{\mid h^{-1}\left(C_{i}\right)}=r_{i}\left(d_{i}-e_{i}\right)
$$

where $e_{i}=1$ or 0 according to whether every center of projection lies on $C_{i}^{\prime}$ or not; and:

$$
\sum_{i} r_{i}\left(d_{i}-e_{i}\right)=4 \text { with } \quad \sum_{i} e_{i} \leqq 1
$$

Now we examine the various possibilities:

If $\operatorname{deg} C^{\prime}=5, h$ is birational: since $g \geqq 6, C$ is a plane quintic.

If $\operatorname{deg} C^{\prime} \leqq 4$ and $C^{\prime}$ is irreducible, one must have $r_{1}=2, d_{1}=3$ : we get case b).

Suppose $\operatorname{deg} C^{\prime} \leqq 4, C^{\prime}$ reducible. If $r_{i}=1$ for some $i, C_{i}^{\prime}$ must have at least 4 intersection points with the rest of $C^{\prime}$, by (A): the only possibility is $d_{1}=d_{2}=2$, $r_{1}=2, r_{2}=1, e_{1}=1$. But then $\operatorname{deg} L_{\mid h^{-1}\left(C_{2}^{\prime}\right)}=2$, which contradicts (B).

Thus one has $r_{i} \geqq 2$ for all $i$; the only possible case is $r_{i}=2$ and $d_{i}-e_{i}=1(i=1,2)$. But then the intersection $h^{-1}\left(C_{1}^{\prime}\right) \cap h^{-1}\left(C_{2}^{\prime}\right)$ contains at most 4 points, and this contradicts either (A) or (B).

(4.9.4) The elimination of possibility (ii) in (4.9.2) is more tedious.

We first suppose $g \geqq 8$. We proceed as in (4.9.3): we fix a non-singular $L_{0}$ in $Z$; then we can choose $(\mathrm{g}-8)$ points $P_{1} \ldots P_{\mathrm{g}-8}$ on $C$ such that:

$M=\omega_{C} \otimes L_{0}^{-1}\left(-\sum P_{i}\right) \quad$ is non-singular and $h^{0}(M)=4$,

$h^{0}\left(M \otimes L^{-1}\right) \geqq 1 \quad$ for any $L$ in $Z$.

We conclude that $M$ defines a morphism $h: C \rightarrow \mathbf{P}^{3}$ such that for $L$ generic in $Z$, the morphism $C \rightarrow \mathbf{P}^{1}$ defined by $L$ is the composition of $h$ with a projection from $\mathbf{P}^{3}$ to $\mathbf{P}^{1}$.

We use the same notation as in (4.9.3):

$$
h(C)=C^{\prime}=\bigcup_{i} C_{i}^{\prime}, \quad d_{i}=\operatorname{deg} C_{i}^{\prime}, \quad r_{i}=\operatorname{deg} h_{\mid h^{-1}\left(C_{i}\right)}
$$


$e_{i}=$ intersection number of $C_{i}^{\prime}$ with a generic line of projection so that

$$
\operatorname{deg} L_{\mid h^{-1}\left(C_{i}\right)}=r_{i}\left(d_{i}-e_{i}\right) \text { and } \sum r_{i}\left(d_{i}-e_{i}\right)=5(\mathrm{C}) \text {. }
$$

One has $e_{i} \leqq 2$ and $\sum_{i} e_{i} \leqq 2$ (if $C^{\prime}$ has a 2-dimensional family of trisecants, it must be the family of lines lying in a plane $\Pi$ containing some component $C_{j}^{\prime}$; but if the generic line of projection lay in $I I$, one would have $d_{j}-e_{j}=0$, which is impossible (4.9.2)).

Now we look at the various possibilities:

i) If $h$ was birational, the curve $C^{\prime}$ would have degree $\leqq 7$ and genus $\geqq 8$. Suppose $C^{\prime}$ does not contain any plane curve of degree $\geqq 3$. Then we can find a plane $\Pi$ in $\mathbf{P}^{3}$ such that $\Pi \cap C^{\prime}$ consists of distinct points $P_{1}, \ldots, P_{d}(d \leqq 7)$, no 3 of them lying on a line. For $r \geqq 3$, we can always find a surface of degree $r$ passing through $P_{1} \ldots P_{k-1}$ and not $P_{k}(k \leqq d)$ : namely, one can find a union of $r$ planes with this property. This implies:

$$
h^{0}\left(C^{\prime}, h^{*} \mathcal{O}_{\mathbf{P}}(r)\right)-h^{0}\left(C^{i}, h^{*} \mathcal{O}_{\mathbf{P}}(r-1)\right)=d \quad \text { for } r \geqq 3 .
$$

Since $h^{1}\left(C^{\prime}, h^{*} \mathcal{O}_{\mathbf{P}}(r)\right)=0$ for $r$ large enough, we get:

$$
h^{1}\left(C^{\prime}, h^{*} \mathcal{O}_{\mathbf{P}}(2)\right)=0 \text {. }
$$

Hence $h^{0}\left(C^{\prime}, h^{*} \mathcal{O}_{\mathbf{P}}(2)\right)=2 d+1-p_{a}\left(C^{\prime}\right) \leqq 7$.

Thus $C^{\prime}$ must be contained in 3 linearly independent quadrics, which is impossible.

If $C^{\prime}$ contains a plane curve of degree $\geqq 3$, one checks easily (using (A)) that $p_{a}\left(C^{\prime}\right) \leqq 6$. Thus $h$ is not birational; $C^{\prime}$ must be reducible, of degree $\leqq 6$.

ii) There cannot be any component of degree 5 by (A) and (B).

iii) There cannot be any component $C_{i}^{\prime}$ of degree 4 : by $(C)$ and $(A)$, one must have $r_{i}=1$; by (B), this implies $e_{i}=2$ and $n=6$, i.e. $C^{\prime}$ is the union of a rational quartic and two trisecants; but this contradicts (A).

iv) If $C_{i}^{\prime}$ is a conic, $e_{i}=1$.

Proof of iv. If $r_{i}=2$, iv) follows from (C) and (A). If $r_{i}=1$, we get from (B) $n=6$; this is seen to be incompatible with (C).

v) There cannot be any component $C_{i}^{\prime}$ of degree 3.

Proof of $\mathrm{v}$. If $e_{i}=2, C^{\prime}$ is the union of $C_{i}^{\prime}$ and some lines (by iv)); one checks that this always contradicts (A). If $r_{i}=1, p_{a}\left(C_{i}^{\prime}\right)=0$, one gets by (B) $n \geqq 6$, which is impossible by (C). If $r_{i}=1, p_{a}\left(C_{i}^{\prime}\right)=1, C^{\prime}$ is a plane curve; by (A) $C^{\prime}$ must be the union of $C_{i}^{\prime}$ and a rational curve having 4 common points, which is impossible. If $r_{i}=2, e_{i}=1$, one gets a contradiction to (A) (using (C)).

vi) Therefore $C^{\prime}$ is a union of lines and conics; moreover $d_{i}-e_{i}=1$ for all $i$, and $\sum r_{i}=5$. One checks easily that every choice for the $\left(r_{i}\right)$ leads to a contradiction with $(\mathrm{A})$ or $(\mathrm{B})$.

(4.9.5) We now suppose $g=7, C$ irreducible. Then we can modify the argument in [M 2, p. 350] as follows: by Riemann-Roch, we get for $L$ generic in $Z: \omega_{C} \otimes L^{-2}$ $\cong \mathcal{O}_{\mathrm{C}}(p+q)$, where $p$ and $q$ are non-singular points of $C$. Let $W$ be the locus of 
effective divisors of degree 2 on $C, C_{\text {reg }}$ the open set of non-singular points of $C$, $d: J C \rightarrow J C$ the multiplication by 2 . Choosing base points, we get embeddings:

$$
C_{\mathrm{reg}} \subset W \subset J C
$$

since the restriction to $C_{\text {reg }}$ of any irreducible covering of $J C$ is irreducible (see for instance [Se, $\S 6$, Prop. 10]), we conclude that $d^{-1}(W)$ is irreducible. Consequently we get as in [M2] $h^{0}(M) \geqq 3$ for any $M$ with $M^{2} \cong \omega_{C}$. But there is always such an $M$ with $h^{\circ}(M)$ even. To see this, we can use Corollary 1.2 if the normalization $N$ of $C$ is not rational; if $N$ is rational, we find an equisingular deformation of $C$ into a hyperelliptic curve $C_{0}$ (i.e. such that there exists a two-sheeted covering $\left.p: C_{0} \rightarrow \mathbf{P}^{1}\right)$, use Theorem 1.1 and the fact that if $M=p^{*} \mathcal{O}_{\mathbf{P}}(3)$, one has $M^{\otimes 2}=\omega_{C_{0}}$ and $h^{0}(M)=4$.

Thus in any case we get an $M$ of degree 6 with $h^{0}(M) \geqq 4$; one checks easily that $M$ is non-singular, hence by Lemma $4.7 \mathrm{C}$ is hyperelliptic.

(4.9.6) Suppose finally $g=7, C$ reducible. Again for $L$ generic in $Z$ we get $\omega_{C} \otimes L^{-2} \cong \mathcal{O}_{C}(p+q)$, where $p, q$ are non-singular points of a component $C_{1}$. We put $C_{0}=\bigcup_{i>1} C_{i}, n=\# C_{0} \cap C_{1}$.

If $p_{a}\left(C_{0}\right)=0$, it turns out that we can still apply the argument of (4.9.5). We first notice that the Jacobian of $C$ is isomorphic to the Jacobian of the irreducible curve obtained from $C_{1}$ by identifying the points of $C_{0} \cap C_{1}$; from this we deduce as in (4.9.5) that the set of line bundles $L$ such that $\omega_{C} \otimes L^{-2} \cong \mathcal{O}_{C}(p+q)$, for some non-singular points $p, q \in C_{1}$, is irreducible. Thus we get $h^{0}(M(-p)) \geqq 2$ for any $M$ with $M^{2} \cong \omega_{C}$ and any $p$ non-singular in $C_{1}$. Now we must rule out the possibility that every section of $M$ vanishes on $C_{1}$; but this is impossible since the kernel of the restriction

$$
H^{0}\left(C, \omega_{\mathcal{C}}\right) \rightarrow H^{0}\left(C_{1}, \omega_{C \mid C_{1}}\right)
$$

is $H^{0}\left(C_{0}, \omega_{C_{0}}\right)$, which is zero. So, we obtain $h^{0}(M) \geqq 3$, and we conclude as in (4.9.5).

Using (A) and (B), we find two cases with $p_{a}\left(C_{0}\right) \neq 0$ :

a) $\operatorname{deg} L_{\mid C_{1}}=1, p_{a}\left(C_{1}\right)=0, n=6$.

b) $\operatorname{deg} L_{\mid C_{1}}=2, p_{a}\left(C_{1}\right)=p_{a}\left(C_{0}\right)=1, n=6$.

We notice that in both cases $L_{\mid C_{0}}$ is fixed (since $\left.L_{\mid C_{0}}^{2} \cong \omega_{C \mid C_{0}}\right)$ and $h^{0}\left(C_{0}, L_{\mid C_{0}}\right)=3$, $h^{0}\left(C_{1}, L_{\mid C_{1}}\right)=2$. Let $g: C_{0} \rightarrow \mathbf{P}^{2}$ be the morphism defined by $L_{\mid C_{0}}$; put $C_{0} \cap C_{1}$ $=\left\{x_{1} \ldots x_{6}\right\}$. A line bundle in $Z$ corresponds to a morphism $h: C_{1} \rightarrow \mathbf{P}^{1}$ (defined by $\left.L_{\mid C_{1}}\right)$, plus a projection $\varphi$ from $\mathbf{P}^{2}$ to $\mathbf{P}^{1}$ such that: $\varphi \circ g\left(x_{i}\right)=h\left(x_{i}\right)(1 \leqq i \leqq 6)$. In case a), $L_{\mid C_{1}}$ is fixed, so any projection from $\mathbf{P}^{2}$ to $\mathbf{P}^{1}$ should conserve the projective relations between the 6 distinct points $g\left(x_{i}\right)$, which is impossible.

In case b), for each degree 2 morphism $h$ there must be a one-dimensional family of projections; this implies that the $g\left(x_{i}\right)$ lies on a conic $Q$, and the center of projection lies on $Q$. But then every morphism $h$ should give the same projective relations between the $h\left(x_{i}\right)$, which is easily seen to be impossible.

We are now in position to prove the main theorem of this section. For simplicity of the statement, we assume that $\tilde{C}$ (or equivalently $C$ ) is a stable curve 
([D-M]); in our situation, this means that we eliminate the case $C=C_{1} \cup C_{2}$, with \# $C_{1} \cap C_{2}=2$ and $C_{1}$ rational (see 4.11 .3 below). We say that a curve is hyperelliptic if it can be realized as a two-sheeted covering of $\mathbf{P}^{1}$.

(4.10) Theorem. Let $\tilde{C}$ be a stable curve of genus $2 g-1, l$ an involution of $\tilde{C}$ satisfying $(*), C=\tilde{C} /(l)$ the quotient curve, $(P, \Xi)$ the associated Prym variety. Recall that $p_{a}(C)=g$ and $\operatorname{dim} P=g-1$; we assume $g \geqq 5$. Then:

If $\operatorname{dim}$ Sing $\Xi=g-3, C$ is hyperelliptic, or $C=C_{1} \cup C_{2}$ with \# $C_{1} \cap C_{2}=2$; $(P, \Xi)$ is a product of two principally polarized abelian varieties.

If $\operatorname{dim} \operatorname{Sing} \Xi=g-4, C$ is hyperelliptic or obtained from a hyperelliptic curve by identifying two points; $(P, \Xi)$ is a hyperelliptic Jacobian.

If $\operatorname{dim} \operatorname{Sing} \Xi=g-5$, one of the following holds:

a) $C$ is a 3-sheeted covering of $\mathbf{P}^{\mathbf{1}}$; then $(P, \Xi)$ is a Jacobian.

b) $C$ is obtained from a hyperelliptic curve by identifying two points; then $(P, \Xi)$ is a Jacobian.

c) $C$ is a double cover of a stable curve of genus one and $g \geqq 6$.

d) $C$ is a genus 5 curve with one vanishing thetanull (that is, a line bundle $N$ such that $\left.h^{0}(N)=2, N^{2} \cong \omega_{c}\right)$ and $h^{0}\left(\tilde{C}, \pi^{*} N\right)$ is even.

e) $C$ is a plane quintic and $h^{\circ}\left(\tilde{C}, \pi^{*} \mathcal{O}_{C}(1)\right)$ is odd.

f) $C$ is obtained from a hyperelliptic curve by identifying two pairs of points.

g) $C$ is obtained from a genus 4 curve with one vanishing thetanull by identifying two points.

h) $C=C_{1} \cup C_{2}$ with \# $C_{1} \cap C_{2}=4$, and neither $C_{1}$ nor $C_{2}$ is a rational curve.

i) $C=C_{1} \cup C_{2}$ with \# $C_{1} \cap C_{2}=4, C_{1}$ is rational and $C_{2}$ is a hyperelliptic curve of genus $\geqq 3$.

j) $C=C_{1} \cup C_{2}$ with $C_{1} \cap C_{2}=\left\{u_{1}, \ldots, u_{4}\right\}, C_{1}$ is rational and $\omega_{C_{2}} \cong \mathcal{O}_{C_{2}}\left(\sum u_{i}\right)$ (hence $p_{a}(C)=6$ ).

Proof. Let $Z$ be an irreducible component of $\operatorname{Sing} \Xi$ with $\operatorname{dim} Z \geqq g-5$, and $L$ a generic line bundle in $Z$. According to Proposition 4.2 we can find two linearly independant sections $s, t$ of $L$ such that $s \otimes l^{*} t=l^{*} s \otimes t$.

(4.10.1) Assume first that the sections $s, t$ have the property that at each singular point of $\tilde{C}$, either $s$ or $t$ is non-zero. Then, by Lemma $4.4, L$ is of the form $\pi^{*} M(E)$; that is, for each $L$ in $Z$ we get:

- a line bundle $M$ on $C$ with $h^{0}(M) \geqq 2$;

- an effective divisor $\pi_{*} E \in\left|\omega_{C} \otimes M^{-2}\right|$.

(Moreover if $E=0$, one has the supplementary condition $h^{0}\left(\pi^{*} M\right)$ even.)

Conversely for any such line bundle $M$ and any effective divisor $D \in\left|\omega_{C} \otimes M^{-2}\right|$ we get as in [M 2] a finite number of points in Sing $\Xi$.

Thus we can bound $\operatorname{dim} Z$ by:

(dimension of possible $M$ 's $)+h^{0}\left(\omega_{c} \otimes M^{-2}\right)-1$.

Assume $C$ has no non-singular $g_{2}^{1}$ and $\operatorname{deg} M \leqq g-2$; then Lemmas 4.7 and 4.9 give $\operatorname{dim} Z \leqq g-5$, and $\operatorname{dim} Z=g-5$ only in cases a) to d) of Lemma 4.9. This 
gives cases a), c), e), h), i), j) of the theorem (for plane quintics, the same study as in $[\mathrm{M} 2, \mathrm{p} .347]$ gives the supplementary condition $h^{0}\left(\tilde{C}, \pi^{*} \mathcal{O}_{C}(1)\right)$ odd $)$. In case a), $(P, \Xi)$ is a Jacobian by $[\mathrm{R}]$.

If $\operatorname{deg} M=g-1, M$ is a theta-characteristic; this can give a $(g-5)$-dimensional Sing $\Xi$ only in genus 5 (case d)).

If $C$ has a non-singular $g_{2}^{1}$, this $g_{2}^{1}$ defines a morphism $g: C \rightarrow \mathbf{P}^{1}$; if at least one component of $C$ is mapped to a point, then $C$ is a union $C_{1} \cup C_{2}$ with $\# C_{1} \cap C_{2}=2$, and we conclude by Lemma 4.11 below. If not, $g$ is a two-sheeted covering, and $C$ is hyperelliptic; at this point one can either extend the analysis of two-sheeted coverings of hyperelliptic curves in [M 2] to singular curves, or simply notice that a singular hyperelliptic curve is a specialization of a nonsingular one, hence the Prym variety must be a hyperelliptic Jacobian or a product of two hyperelliptic Jacobians.

(4.10.2) Next suppose that the two sections $s, t$ of $L$ are such that $s$ and $t$ do not vanish simultaneously on any component of $\tilde{C}$, but vanish simultaneously at some singular points $z_{1}, \ldots, z_{n}$. Let $f: \tilde{D} \rightarrow \tilde{C}$ be the normalization of $\tilde{C}$ at $z_{1}, \ldots, z_{n}, D=\tilde{D} /(l)$ the quotient curve, $\pi^{\prime}: \tilde{D} \rightarrow D$ the projection; we define: $L_{1}=f^{*} L\left(-\sum\left(x_{i}+y_{i}\right)\right)$, where $\left\{x_{i}, y_{i}\right\}=f^{-1}\left(z_{i}\right)$. Then $N m\left(L_{1}\right) \cong \omega_{D}$, and since $s$ and $t$ define global sections of $L_{1}, L_{1}$ has property (P).

First suppose that $\tilde{D}$ is connected. Then we can bound the dimension of possible $L_{1}$ 's as in 4.10 .1 ; but to a line bundle $L_{1}$ on $\tilde{D}$ corresponds only a finite number of $L$ 's in the Prym variety (see Remark 3.6). Since $p_{a}(D)=g-n$, the only possibility in order to get $\operatorname{dim} Z \geqq g-5$ is $n=2$ and $D=D_{1} \cup D_{2}$ with \# $D_{1} \cap D_{2}=2$, which gives case $h$ ), or $n=2$ and $D$ hyperelliptic, which gives case $f$ ), or $n=1$ and $D$ hyperelliptic. In the last situation we can have $\operatorname{dim} Z=g-5$ or $g-4$; but both cases are specializations of case a). Namely, put $C^{\prime}=D \cup R$, where $R$ is a rational curve and $D \cap R=\left\{\pi^{\prime}\left(x_{1}\right), \pi^{\prime}\left(y_{1}\right)\right\}$. We can find a one-dimensional family of nonsingular trigonal curves, with $C^{\prime}$ as special fibre; then we blow down $R$ and get $C$ as special fibre. It follows that the Prym variety is a Jacobian, which must be hyperelliptic if $\operatorname{dim} \operatorname{Sing} \Xi=g-4$.

Now suppose that $\tilde{D}$ is disconnected: say $\tilde{D}=\tilde{D}_{1} \cup \tilde{D}_{2}$, with $\tilde{D}_{1} \cap \tilde{D}=\emptyset$. The hypothesis on $s, t$ implies $h^{0}\left(L_{1 \mid \tilde{D}_{1}}\right) \geqq 1, h^{0}\left(L_{1 \mid \tilde{D}_{2}}\right) \geqq 1$. Conversely, this is enough to insure that $L_{1}$ has property (P)! Namely take $a \in H^{0}\left(D_{1}, L_{1 \mid \tilde{D}_{1}}\right), b \in H^{0}\left(D_{2}, L_{1 \mid \tilde{D}_{2}}\right)$, and define $s=(a, 0), t=(0, b)$ via the identification:

$$
H^{0}\left(\tilde{D}, L_{1}\right)=H^{0}\left(\tilde{D}_{1}, L_{1 \mid \tilde{D}_{1}}\right) \oplus H^{0}\left(\tilde{D}_{2}, L_{1 \mid \tilde{D}_{2}}\right)
$$

then clearly $l^{*} s \otimes t=s \otimes \imath^{*} t=0$.

Let $D_{i}=\pi^{\prime}\left(\tilde{D}_{i}\right)$; the locus of effective divisors $E$ on $\tilde{D}_{1}$ such that $\pi_{*}^{\prime} E \in\left|\omega_{D_{1}}\right|$ has dimension $p_{a}\left(D_{1}\right)-1$. Thus we get:

$$
\operatorname{dim} Z \leqq p_{a}\left(D_{1}\right)-1+p_{a}\left(D_{2}\right)-1=g-n-1
$$

provided neither $D_{1}$ nor $D_{2}$ is rational. To get $\operatorname{dim} Z \geqq g-5$, we must have either $n=2$, which gives the case $C=C_{1} \cup C_{2}, \# C_{1} \cap C_{2}=2$, to which we apply Lemma 4.11 below; or $n=4$, which gives case h).

(4.10.3) Finally let us consider the general case. Then it may happen that $s$ and $t$ 
vanish simultaneously on some components of $\tilde{C}$; let $\tilde{F}$ be the union of these components, $\tilde{G}$ the union of the other components, $F=\tilde{F} /(l), G=\tilde{G} /(t), \tilde{F} \cap \tilde{G}$ $=\left\{x_{1} \ldots x_{r}\right\}$. Put $L_{1}=L_{\mid \tilde{G}}\left(-\sum x_{i}\right)$; then, $N m L_{1} \cong \omega_{G}$ and again $L_{1}$ has property (P). Conversely given $L_{1}$ and any line bundle $N$ on $F$ such that $N m N \cong \omega_{C \mid F}$, we get a finite number of line bundles $L$ in $Z$ such that:

$$
L_{\mid \tilde{G}} \cong L_{1}\left(\sum x_{i}\right), \quad L_{\mid \tilde{F}} \cong N .
$$

The dimension of possible $N$ 's is:

$$
p_{a}(\tilde{F})-p_{a}(F)=p_{a}(F)-1+\frac{r}{2}
$$

(recall that we use the convention that $p_{a}(F)=1-\chi\left(\mathcal{O}_{F}\right)$ for a not necessarily connected curve $F$ ). Assume first that $\tilde{G}$ is connected; then we can bound the dimension of possible $L_{1}$ 's as in (4.10.2) by $p_{a}(G)-e$, where $e \geqq 3$. Thus, we get:

$$
\operatorname{dim} Z \leqq p_{a}(G)-e+p_{a}(F)-1+\frac{r}{2}=g-e-\frac{r}{2} .
$$

The only new possibility is $r=4, e=3$ which gives case i), Finally if $\tilde{G}$ is disconnected, the dimension of possible $L_{1}$ 's is bounded by $p_{a}(G)-1$ (4.10.2). Therefore:

$$
\operatorname{dim} Z \leqq p_{a}(G)-1+p_{a}(F)-1+\frac{r}{2}=g-1-\frac{r}{2} .
$$

But, this gives no new case. Thus the proof of the theorem is complete, once we prove the following lemma:

(4.11) Lemma. Suppose $\tilde{C}=\tilde{C}_{1} \cup \tilde{C}_{2}$, with $\tilde{C}_{1} \cap \tilde{C}_{2}=\{p, q\}$. Let $\tilde{C}_{i}^{\prime}(i=1,2)$ be the curve obtained from $\tilde{C}_{i}$ by identifying $p$ and $q, P_{i}$ the Prym variety associated to $\tilde{C}_{i}^{\prime}$ with the involution induced by 1 . Then $P \cong P_{1} \times P_{2}$ as principally polarized abelian varieties.

(4.11.1) We first prove the following more general statement:

- Let $\tilde{C}$ be a curve with ordinary double points, $\imath$ an involution of $\tilde{C}$ satisfying $(*)$, $(P, \Xi)$ the associated Prym variety; let $\tilde{N}$ be a curve obtained from $\tilde{C}$ by blowing-up some singular points, such that $\operatorname{dim} J \tilde{N}=\operatorname{dim} J \tilde{C}-1$. Define $R=\operatorname{Ker}(N m) \subset J \tilde{N}$. Then $R$ is an abelian variety, the principal polarization on $J \tilde{N}$ induces twice a principal polarization $\Psi$ on $R$, and $(R, \Psi)$ is isomorphic to $(P, \Xi)$.

Proof of (4.11.1). As in Proposition 3.5, we get an exact sequence:

$$
0 \rightarrow \mathrm{Z} /(2) \rightarrow \mathrm{Z} /(2) \times P \stackrel{g}{\rightarrow} R \rightarrow 0
$$

where $g: P \rightarrow R$ is an isomorphism. Then the proof of Theorem 3.7 applies to this situation and gives the statement about polarizations.

(4.11.2) Proof of the Lemma. Let $R_{i}=\operatorname{Ker}\left(N m_{\mid J \tilde{C}_{i}}\right)(i=1,2)$. By (4.11.1), the principal polarization on $J \tilde{C}_{i}$ induces twice a principal polarization $\Psi_{i}$, and 
$\left(R_{i}, \Psi_{i}\right)$ is isomorphic to $P_{i}$ with its principal polarization; then by (4.11.1) again, $P$ is isomorphic to the product $\left(R_{1}, \Psi_{1}\right) \times\left(R_{2}, \Psi_{2}\right)$, hence the result.

(4.11.3) The proof of the lemma applies when $\tilde{C}_{2}$, say, is a rational curve: in that case one finds $P \cong P_{1}$. This allows to extend the theorem to non-stable curves (with ordinary double points).

\section{Exchanged Components}

We shall need to study the Prym variety under a more general assumption than hypothesis $(*)$ in $\S 3$. We start from a connected curve $\tilde{C}$ of genus $2 g-1$, with ordinary double points, and an involution $t: \tilde{C} \rightarrow \tilde{C}$. If $C$ is the quotient curve, the norm defines a morphism $N m: . J \tilde{C} \rightarrow J C$, and we define the Prym variety $P$ by $P=(\operatorname{Ker} N m)^{0}$. It is a group variety, extension of an abelian variety by a torus.

We now assume:

(**) $\left\{\begin{array}{l}-l \text { is not the identity on any component of } \tilde{C} . \\ -p_{a}(C)=g . \\ -P \text { is an abelian variety. }\end{array}\right.$

Let us fix some notation:

$n_{f}=\#$ nodes of $\tilde{C}$ fixed under $l$, with the 2 branches not exchanged.

$n_{f}^{\prime}=\#$ nodes of $\tilde{C}$ fixed under $t$, with the 2 branches exchanged.

$2 n_{e}=\#$ nodes exchanged under $t$.

$c_{f}=\#$ components fixed under $\boldsymbol{l}$.

$2 c_{e}=\#$ components exchanged under 1 .

$r=\#$ fixed non-singular points of $t$.

(5.1) Lemma. The assumptions (**) are equivalent to $r=n_{f}^{\prime}=0, n_{e}=c_{e}$.

Proof. Let $\tilde{N}$ (resp. $N$ ) be the normalization of $\tilde{C}$ (resp. C). The covering $\pi^{\prime}: \tilde{N} \rightarrow N$ is ramified at the points of $\tilde{N}$ lying over fixed non-singular points, and fixed singular points with no exchanged branches; hence, by the Hurwitz formula:

$$
p_{a}(\tilde{N})-1=2\left(p_{a}(N)-1\right)+\frac{r}{2}+n_{f}
$$

so

$$
\begin{aligned}
p_{a}(\tilde{C})-1 & =p_{a}(\tilde{N})-1+2 n_{e}+n_{f}+n_{f}^{\prime} \\
& =2\left(p_{a}(N)-1\right)+\frac{r}{2}+2 n_{e}+2 n_{f}+n_{f}^{\prime} .
\end{aligned}
$$

The singular points of $C$ come from singular points on $\tilde{C}$ with no exchanged branches and from exchanged singular points, hence:

$$
p_{a}(C)-1=p_{a}(N)-1+n_{f}+n_{e} .
$$

Therefore

$$
p_{a}(\tilde{C})-1=2\left(p_{a}(C)-1\right)+\frac{r}{2}+n_{f}^{\prime} .
$$


Thus the condition $p_{a}(C)=g$ is equivalent to $r=n_{f}^{\prime}=0$.

We now express the condition that $P$ is an abelian variety; from the diagram:

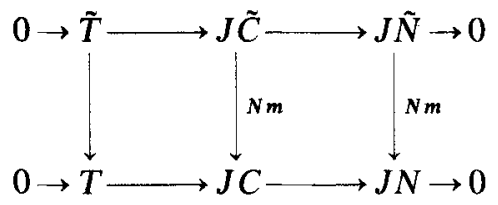

and from the surjectivity of the norm it follows that $P$ is an abelian variety if and only if $\operatorname{dim} \tilde{T}=\operatorname{dim} T$.

Here $\operatorname{dim} \tilde{T}=2 n_{e}+n_{f}-2 c_{e}-c_{f}+1$

$\operatorname{dim} T=n_{e}+n_{f}-c_{e}-c_{f}+1$

thus $\operatorname{dim} \tilde{T}-\operatorname{dim} T=n_{e}-c_{e}$, and the lemma is proved.

So the assumptions (**) are equivalent to (*) if $c_{e}=0$. When $c_{e}=n_{e} \neq 0$, let $B$ be the union of the components of $\tilde{C}$ fixed under $l$; we write $\tilde{C}=A \cup A^{\prime} \cup B$, where $A^{\prime}=\imath(A)$ and $A$ and $A^{\prime}$ have no common component. Recall that to the curve $C$ we associate a graph $\Gamma$ : the vertices of $\Gamma$ are the irreducible components of $\Gamma$, and the edges between two vertices are the intersection points of the two corresponding components. We say that $C$ is tree-like if its graph is a tree and if each irreducible component of $C$ is non-singular.

(5.2) Proposition. (i) If $B=\emptyset$, then $\tilde{C}=A \cup A^{\prime}$ where $A$ can be chosen connected

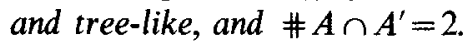

(ii) If $B \neq \emptyset$, then $A \cap A^{\prime}=\varnothing$; each connected component of $A$ is tree-like and meets $B$ at only one point. $B$ is connected and satisfies condition (*).

We shall use the following easy lemma:

(5.3) Lemma. Let $\Gamma$ be a connected graph, $t$ an involution of $\Gamma$ without fixed points. There exists a connected subgraph $S$ of $\Gamma$ such that $S \cap \imath S=\emptyset$ and $S \cup \imath S$ contains every vertex of $\Gamma$.

(Hint: take a connected subgraph $S$ maximal for the property $S \cap t S=\emptyset$.)

Proof of the Proposition. If $B=\emptyset$, we write $\tilde{C}=A \cup A^{\prime}$, where $A$ corresponds to the subgraph $S$ in Lemma 5.3. Let $v$ be the number of vertices of $S, e$ the number of edges, $s$ the number of singular points on $A$ which belong to only one component. The equality $c_{e}=n_{e}$ gives:

$$
v=e+s+\frac{1}{2}\left(\# A \cap A^{\prime}\right) \text {. }
$$

Since $1-v+e \geqq 0$, we get $s=0, \# A \cap A^{\prime}=2$ and $1-v+e=0$, that is $A$ is tree-like.

Assume $B \neq \varnothing$. By Lemma 5.1, the points of $A \cap A^{\prime}$ are exchanged under 1 ; we put:

$\# A \cap A^{\prime}=2 r$,

$\# A \cap B=\# A^{\prime} \cap B=m$.

$i_{A}=\#$ irreducible components of $A$. 
$c_{A}=\#$ connected components of $A$.

$n_{A}=$ \# singular points of $A$.

$2 b=\#$ singular points of $B$ exchanged under $t$.

Then $c_{e}=i_{A}, n_{e}=n_{A}+r+m+b$.

Recall that for any curve $A, n_{A}-i_{A}+c_{A} \geqq 0$ (this is the first Betti number of the graph of $A$, plus the number of double points of $A$ which belong to only one component).

Thus, if $c_{e}=n_{e}$ :

$$
0=n_{A}-i_{A}+r+m+b \geqq r+m+b-c_{A} .
$$

Since $\tilde{C}$ is connected, any component of $A \cup A^{\prime}$ meets $B$. But one can choose $A$ in such a way that a connected component of $A \cup A^{\prime}$ is either a connected component of $A$ or $A^{\prime}$, or can be written $D \cup i D$ where $D$ is connected (by Lemma 5.3). From this, we get $m \geqq c_{A}$, hence:

$$
0 \geqq r+m+b-c_{A} \geqq r+b \geqq 0
$$

and $r=0, m=c_{A}, n_{A}-i_{A}+c_{A}=0, b=0$, which gives the proposition.

(5.4) Theorem. Under the assumptions (**), any theta divisor of $J \tilde{C}(\S 2)$ induces on $P$ twice a principal polarization $\Xi$. In case (i) of Proposition $5.2,(P, \Xi)$ is isomorphic to the Jacobian variety $J A$ (with its principal polarization); in case (ii), $(P, \Xi)$ is isomorphic to the product $J A \times Q$ (with the product polarization), where $Q$ is the Prym variety associated to $(B, \imath)$.

Proof. We recall that if $\left(A, \Theta_{A}\right),\left(B, \Theta_{B}\right)$ are two principally polarized abelian varieties, the divisor $\Theta_{A} \times B+A \times \Theta_{B}$ defines a principal polarization on $A \times B$, which we call the product polarization.

In case (ii), $J \tilde{C}$ is isomorphic to $J A \times J A \times J B$, and $P=(\operatorname{Ker} N m)^{0}$ to the subvariety $j(J A) \times Q$, where $j$ is the embedding of $J A$ in $J A \times J A$ defined by $j(x)$ $=(x,-x)$ (note that since $A$ is tree-like, $J A$ is an abelian variety). If $B^{\prime}$ is the normalization of $B$, the polarization induced on $P$ by a theta divisor of $J \tilde{C}$ is the pull-back of the product polarization on $J A \times J A \times J B^{\prime}$ (by Proposition 2.2); therefore, it is the product of the polarization on $j(J A)$ induced by the product polarization of $J A \times J A$, and the polarization on $Q$ induced by a theta divisor of $J B$. The result follows by noting that the product polarization on $J A \times J A$ induces twice the principal polarization on $j(J A)$.

In case (i), $\tilde{C}$ is obtained from the disjoint union $A \coprod A^{\prime}\left(A^{\prime}=A\right)$ by identifying a point $p$ (resp. $q$ ) in $A$ with a point $q$ (resp. p) in $A^{\prime} ; C=\tilde{C} / l$ is obtained from $A$ by identifying $p$ and $q$, and $\pi: \tilde{C} \rightarrow C$ is an unramified two-sheeted covering.

We look at the exact sequences:

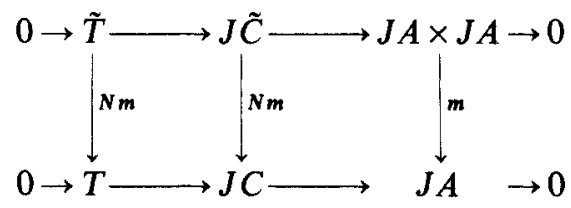


here $m$ is the addition morphism; $\tilde{T}$ and $T$ are isomorphic to the multiplicative group of $k$. One checks immediately that $N m: \tilde{T} \rightarrow T$ is an isomorphism; hence $P \cong \operatorname{Ker} m=j(J A)$, and by the preceding remark the polarization induced on $P$ is twice the principal polarization of the Jacobian variety $J A$.

\section{Compactification of the Prym Mapping}

In this section we apply the results of [D-M] to "compactify" the mapping which associates to a curve plus a 2-sheeted covering the corresponding Prym variety. The natural language here is the theory of stacks, used in [D-M]. However, to avoid excessive technicality, we first prove the main result over $\mathbf{C}$, with a more down-to-earth language; then we consider the situation over $\mathbf{Z}$, using algebraic stacks.

(6.1) Construction. There exists an irreducible complete variety $\bar{S}$ over $k$, a family of stable curves $q: \tilde{\mathscr{C}} \rightarrow \bar{S}$, and a $\bar{S}$-involution $t: \tilde{\mathscr{C}} \rightarrow \tilde{\mathscr{C}}$ such that:

a) For each $s$ in $\bar{S}$, the induced involution $\imath_{s}: \tilde{\mathscr{C}}_{s} \rightarrow \tilde{\mathscr{C}}_{s}$ is different from the identity on each component of $\tilde{\mathscr{C}}_{s}$.

b) $\tilde{\mathscr{C}}_{s}$ has genus $2 \mathrm{~g}-1$, and the quotient curve $\tilde{\mathscr{C}}_{s} /\left(l_{s}\right)$ has genus $g$.

c) For any non-singular curve $C$ of genus $2 g-1$ with a fixed point free involution $l$, the pair $(\tilde{C}, l)$ is isomorphic to $\left(\tilde{\mathscr{C}}_{s}, l_{s}\right)$ for some $s$ in $\bar{S}$.

We start with a complete family of stable curves of genus $2 g-1$

$q: \tilde{X} \rightarrow T$,

where $T$ is a complete, irreducible variety ([D-M]). Using [D-M, p. 84], we see that the functor of $T$-involutions of $\tilde{\mathscr{X}}$ is representable by a scheme finite over $T$; this means that we can find a complete variety $I$, a finite morphism $r: I \rightarrow T$, and an $I$-involution $\sigma$ of $\tilde{\mathscr{X}} \times{ }_{T} I$, such that for any $t$ in $T$, the correspondence:

$r^{-1}(t) \rightarrow\left\{\right.$ involutions of $\left.\tilde{\mathscr{X}}_{t}\right\}$

$$
x \mapsto \sigma_{\mid \bar{x}_{\varepsilon} \times(x)}
$$

is one-to-one.

Since the moduli space of non-singular curves of genus $g$ with a two-sheeted covering is irreducible ([D-M]), we can find an irreducible component $\bar{S}$ of $I$ such that property c) is satisfied, where we denote by $(\tilde{\mathscr{C}}, l)$ the pull-back of $\left(\tilde{\mathscr{X}} \times_{T} I, \sigma\right)$ over $\bar{S}$.

Suppose $\imath$ equals the identity on some component $D$ of $\tilde{\mathscr{C}}_{s}$. One can find an open set $\tilde{U}$ in $\tilde{\mathscr{C}}$, stable under $l$, smooth over $\bar{S}$, such that $\tilde{U} \cap D \neq \varnothing$. The quotient $U=\tilde{U} /(l)$ is smooth over $\bar{S}$, hence the finite morphism $\pi: \tilde{U} \rightarrow U$ is flat (use for instance [EGA IV 11.3.11+15.4.2]). Since $\pi$ has degree 2 , it must be ramified along $\tilde{U} \cap D$, which is impossible since $D$ is reduced. This gives a); since the quotient curve $\tilde{\mathscr{C}}_{s} /\left(l_{s}\right)$ is reduced, its genus is independant of $s$, hence equal to $g$.

(6.2) We put $\mathscr{C}=\tilde{\mathscr{C}} /(\mathrm{l})$. By a result of M.Artin ([A]), the Jacobians of the curves $\tilde{\mathscr{C}}_{s}\left(\right.$ resp. $\left.\mathscr{C}_{s}\right)$ fit together in an algebraic space over $\bar{S}$, denoted by $\underline{\operatorname{Pic}}^{0}(\tilde{\mathscr{C}} / \bar{S})$ 
(resp. $\left.\underline{\operatorname{Pic}}^{0}(\mathscr{C} / \bar{S})\right)$. The norm defines a morphism:

$N m: \underline{\operatorname{Pic}}^{0}(\tilde{\mathscr{C}} / \bar{S}) \rightarrow \underline{\operatorname{Pic}}^{0}(\mathscr{C} / \bar{S})$.

Let $\overline{\mathscr{P}}=(\operatorname{Ker} N m)^{0}$. It follows from the general theory of group schemes (e.g. [SGA 3 exp. $\mathrm{VI}_{\text {B }}$ Cor. 4.4]) that $\overline{\mathscr{P}}$ is a smooth algebraic space over $\bar{S}$, whose fibre over $s$ is the Prym variety associated to $\left(\tilde{\mathscr{C}}_{s}, l\right)$.

The set $S$ of points $s$ in $\bar{S}$ such that $\overline{\mathscr{P}}_{s}$ is an abelian variety is open. By Lemma 1.3 in [FGA exp. 236], the restriction $\mathscr{P}$ of $\overline{\mathscr{P}}$ to $S$ is proper over $S$.

Moreover, according to Remark 2.4, by choosing a line bundle $L$ on $\tilde{\mathscr{C}}$ such that $\operatorname{deg}\left(L_{s}\right)=2 \mathrm{~g}-2$ and $h^{0}\left(L_{s}\right)=0$ for each $s$, we can define (locally on $S$ for the étale topology) a divisor $\Theta$ over $\underline{\operatorname{Pic}}^{0}(\tilde{C} / S)$, and the restriction of $\Theta$ to $\mathscr{P}$ gives a $S$-morphism:

$$
\rho: \mathscr{P} \rightarrow \hat{\mathscr{P}}
$$

such that $\rho=2 \rho^{\prime}$, where $\rho^{\prime}$ is an isomorphism from $\mathscr{P}$ onto $\widehat{\mathscr{P}}$ (by Theorems 3.7 and 5.4).

Since $\rho$ does not depend on the choice of $L$, the polarization $\rho^{\prime}$ is defined globally over $S$, so that we get a flat family of principally polarized abelian varieties over $S$, hence a morphism:

$$
p: S \rightarrow \mathscr{A}_{\mathrm{g}-1}
$$

where $\mathscr{A}_{\mathrm{g}-1}$ is the (coarse) moduli space of principally polarized abelian varieties of dimension $(g-1)$ over $k$ (see [M 4]. Over $\mathbf{C}, \mathscr{A}_{\mathrm{g}}$ is the quotient of the Siegel upper half-space $H_{g}$ by the modular group $\operatorname{Sp}(2 g, \mathbf{Z})$ ).

(6.3) Proposition. The mapping $p$ is proper.

Proof. Using the valuative criterion of properness ([EGA II, 7.3.8]) and the completeness of $\bar{S}$, we are reduced to prove the following:

Let $T$ be the spectrum of a complete discrete valuation ring, $\eta$ its generic point; also let $\tilde{\mathscr{C}} \rightarrow T$ be a family of stable curves with involution, such that $\left(\tilde{\mathscr{C}}_{\eta}, l\right)$ satisfies condition $(* *)$ and the Prym variety $\mathscr{P}_{\eta}$ has good reduction (i.e. extends to an abelian variety over $T$ ). Then $\mathscr{P}_{s}$ is an abelian variety.

But now since $\mathscr{P}_{s}$ is an extension of an abelian variety by a torus, it is isomorphic to the neutral component of the Neron model of $\mathscr{P}_{\eta}$ over $T$ ([SGA 7 IX, 3.2]) which is abelian by hypothesis. Hence $p$ is proper.

(6.4) Proposition. Assume $k=\mathrm{C}$. Then every principally polarized abelian variety of dimension $\mathrm{g} \leqq 5$ is a (generalized) Prym variety.

Proof. In view of Proposition 6.3 and of the irreducibility of $\mathscr{A}_{\mathrm{g}-1}$, it suffices to prove that $p$ is generically surjective, and this is a classical result by Wirtinger ([W, §59]). A different proof of Wirtinger's theorem can be given as follows: using Theorem 5.4 one sees easily that every Jacobian variety is a specialization of a Prym variety, hence $p(S)$ contains the Jacobians. This gives the result for $g \leqq 3$; and also for $g=4$, since $p(S)$ is irreducible, contains the divisor of Jacobians and some other abelian varieties. 
In genus 5 , it is enough to prove that for a suitable choice of $S$, the morphism $p$ is unramified at some point $s$ of $S$. If $C=\mathscr{C}_{s}$ is non-singular, this is easily seen to be equivalent to the following: the natural map:

$$
\varphi: S^{2} H^{0}\left(C, \omega_{C} \otimes \eta\right) \rightarrow H^{0}\left(C, \omega_{C}^{\otimes 2}\right)
$$

(where $\eta$ is the line bundle with $\eta^{2}=\mathscr{O}_{C}$ associated to the 2-sheeted covering $\pi: \tilde{C} \rightarrow C)$ is an isomorphism.

We start from a non-singular curve $X$ of genus 5 , not trigonal, without vanishing theta-nulls. $X$ is a complete intersection of 3 quadrics $(P),(Q),(R)$ in $\mathrm{P}^{4}$; we identify the set of quadrics $(\lambda P+\mu Q+\nu R)$ containing $X$ to a projective plane $\Pi$. Inside of $\Pi$ we consider the discriminant locus $C$, which is a non-singular curve of degree 5 (hence of genus 6). The points of $C$ correspond to quadrics of rank 4 through $X$; these quadrics contain two systems of generatrices, which define an unramified 2-sheeted covering of $C$, hence a line bundle $\eta$ on $C$ with $\eta^{2} \simeq \mathcal{O}_{c}$.

Besides the embedding $i: C \rightarrow I I$, we consider an other embedding $j: C \rightarrow \mathbf{P}^{4}$ defined by:

$j(x)=$ focus of the singular quadric corresponding to $x$. One checks that: $j^{*} \mathcal{O}_{\mathbf{p}}(1)=i^{*} \mathcal{O}_{n}(2) \otimes \eta$.

Now in the product embedding $C \stackrel{(i, j)}{\longrightarrow} \Pi \times \mathbf{P}^{4}, C$ is a complete intersection: in fact it is defined by the 5 equations:

$$
\lambda P_{X_{i}}^{\prime}+\mu Q_{X_{j}}^{\prime}+\nu R_{X_{i}}^{\prime}=0 ; \quad i=0, \ldots, 4 .
$$

Therefore if $p$ and $q$ are the projections from $I \times \mathbf{P}^{4}$ onto $\Pi$ and $\mathbf{P}^{4}$, and $E=\left[p^{*} \mathcal{O}_{I I}(1) \otimes q^{*} \mathcal{O}_{\mathbf{P}}(1)\right]^{5}$, we get a resolution of $\mathcal{O}_{C}$ by the Koszul complex:

$$
0 \rightarrow \Lambda^{5} E \rightarrow \cdots \rightarrow E \rightarrow \mathcal{O}_{\Pi \times \mathbf{P}^{4}} \rightarrow \mathcal{O}_{C} \rightarrow 0 .
$$

Taking tensor products with $q^{*} \mathcal{O}_{\mathbf{p}}(2)$, we conclude by standard arguments that the restriction map:

$$
j^{*}: H^{0}\left(\mathbf{P}^{4}, \mathcal{O}_{\mathbf{P}}(2)\right) \rightarrow H^{0}\left(C, \omega_{\mathrm{c}}^{2}\right)
$$

is an isomorphism; but this map can be identified with $\varphi$, hence the result.

Now we return to the case of an algebraically closed field $k$ of arbitrary characteristic $\neq 2$.

(6.5) Theorem. (i) Every principally polarized abelian variety of dimension $g \leqq 5$ is a (generalized) Prym variety.

(ii) The moduli space $\mathscr{A}_{\mathrm{g}}$ of principally polarized abelian varieties over $k$ is irreducible for $\mathrm{g} \leqq 5$.

Proof. We refer to [D-M] for the definition and the properties of algebraic stacks. All the schemes and stacks we consider are defined over $B=\operatorname{Spec}\left(Z\left[\frac{1}{2}\right]\right)$.

(6.5.1) We start with the algebraic stack $\mathscr{M}_{2 \mathrm{~g}-1}\left[\frac{1}{2}\right]$ classifying stable curves of genus $(2 g-1)$. The stack $\mathscr{M}_{2 g-1}\left[\frac{1}{2}\right]$ is proper over $B$.

According to [D-M, Th. 1.11], the stack $\mathscr{I}^{\prime}$ which classifies stable curves of 
genus $(2 g-1)$ with a non trivial involution is finite and unramified over $\mathscr{M}_{2 \mathrm{~g}-1}\left[\frac{1}{2}\right]$, hence proper over $B$. We consider the subset $\bar{I}$ of $\mathscr{I}^{\prime}$ which classifies curves with involution $(\tilde{C}, \imath) \rightarrow T$ such that for each $t$ in $T$ :

- The involution $l_{t}$ induced on $\tilde{C}_{t}$ is different from the identity on each component of $\tilde{C}_{t}$;

$-p_{a}\left(\tilde{C}_{t} /\left(l_{t}\right)\right)=g$.

The argument of (6.1) shows that $\overline{\mathscr{I}}$ is an open and closed subset of $\mathscr{I}^{\prime}$.

We define as in (6.2) the open subset $\mathscr{I}$ of $\bar{I}$ classifying stable curves with involution such that the associated Prym variety is an abelian variety. We get a morphism of stacks over $B$ :

$p: \mathscr{I} \rightarrow \mathscr{A}_{g-1}^{\prime}$

where $\mathscr{A}_{\mathrm{g}-1}^{\prime}$ denotes the algebraic stack classifying principally polarized abelian varieties of dimension $(g-1)$ over $B$.

(6.5.2) Lemma. $p$ is proper, and surjective if $g \leqq 6$.

Proof. The proof of Proposition 6.3 gives the properness of $p$. Since the restriction of $p$ in characteristic zero is surjective (6.4), $p$ is surjective.

This proves part (i) of the Theorem; it remains to prove that the fibres of $\mathscr{I}$ over $B$ are irreducible. By associating to a curve with involution $(\tilde{C}, \imath)$ the quotient curve $\tilde{C} /(l)$ we get a morphism of algebraic stacks over $B$ :

$q: \mathscr{I} \rightarrow \mathscr{M}_{\mathrm{g}}\left[\frac{1}{2}\right]$.

The morphism $q$ is not representable since the involution $l$ is an automorphism of $(\tilde{C}, l)$ which induces the identity on $\tilde{C} /(l)$. We introduce the universal curve $\tilde{\mathscr{C}}$ (resp. $\mathscr{C}$ ) over $\overline{\mathscr{I}}$ (resp. $\mathscr{M}_{\mathrm{g}}\left[\frac{1}{2}\right]$ ), and its smooth open subset $\tilde{\mathscr{C}}_{\text {reg }}$ (resp. $\mathscr{C}_{\text {reg }}$ ); the stack $\tilde{\mathscr{C}}_{\text {reg }}$ classifies curves with involution $(\tilde{C}, l) \rightarrow T$, plus a section $\tilde{e}: T \rightarrow \tilde{C}_{\text {reg }}$ (we denote by $\tilde{C}_{\text {reg }}$ the open subset of $\tilde{C}$ consisting of points smooth over $T$ ). The stack $\mathscr{C}_{\text {reg }}$ classifies stable curves of genus $g: C \rightarrow T$, plus a section $e: T \rightarrow C_{\text {reg }}$. There is a morphism:

$$
r: \tilde{\mathscr{C}}_{\text {reg }} \rightarrow \mathscr{C}_{\text {reg }}
$$

such that the diagram:

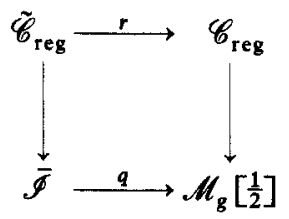

is commutative.

(6.5.3) Lemma. The morphism $r$ is representable and finite.

Proof. This means the following:

a) Let $(\tilde{C}, \imath) \rightarrow T$ be a curve with involution (classified by $\overline{\mathscr{I}}$ ), $\tilde{e}: T \rightarrow \tilde{C}_{\mathrm{reg}}$ a 
section. Then, any $T$-automorphism of $\tilde{C}$ which commutes with $l$, induces the identity on $\tilde{C} /(l)$ and leaves $\tilde{e}$ fixed is the identity.

b) Let $C \rightarrow T$ be a stable curve of genus $g, e: T \rightarrow C_{\text {reg }}$ a section. We consider the functor $F$ which associates to each $T$-scheme $U$ the set:

$$
\begin{gathered}
F(U)=\text { set of isomorphism classes of }\left\{\begin{array}{l}
- \text { stable curves with } \\
\text { involution }(\tilde{C}, t) \rightarrow U \\
+ \text { section } \tilde{e}: U \rightarrow \tilde{C}_{\text {reg }} \\
+U \text {-morphism } \pi: \tilde{C} \rightarrow C_{U}
\end{array}\right\} \\
\text { such that: }\left\{\begin{array}{l}
-(\tilde{C}, l) \text { is classified by } \overline{\mathscr{T}} \\
-\pi \circ \tilde{e}=e \\
-\pi \circ l=\pi \\
-\pi \text { induces an isomorphism } \tilde{C} /(l) \sim C_{V} .
\end{array}\right.
\end{gathered}
$$

Then, $F$ is representable by a scheme finite over $T$.

To prove a), we note that the only non-trivial $T$-automorphism of $\tilde{C}$ which commutes with $t$ and induces the identity on $\tilde{C} /(t)$ is $t$ itself; but by Lemma 5.1, any fixed point of $t$ is singular in its fibre, so $l$ cannot leave $\tilde{e}$ fixed.

Let us prove b). We associate to the data $(\tilde{C}, l, \tilde{e}, \pi)$ the coherent sheaf $\mathscr{L}$ on $C_{U}$ defined by:

$$
\pi_{*} \mathcal{O}_{\tilde{\mathbf{C}}}=\mathcal{O}_{C_{U}} \oplus \mathscr{L}
$$

$\mathscr{L}$ induces on each fibre $\left(C_{U}\right)_{u}$ a torsion-free rank one coherent sheaf. Note that the algebra structure on $\pi_{*} \mathcal{O}_{\tilde{C}}$ gives an isomorphism:

$$
\lambda: \mathscr{L} \longrightarrow \operatorname{Hom}\left(\mathscr{L}, O_{\boldsymbol{C}_{U}}\right) .
$$

The section $\tilde{e}$ corresponds to an isomorphism:

$$
\alpha: e^{*} \mathscr{L} \stackrel{\sim}{\longrightarrow} \mathcal{O}_{\mathbf{v}}
$$

such that $\left(e^{*} \lambda\right)\left(\alpha^{-1}(1)\right)=\alpha$.

Conversely given $(\mathscr{L}, \alpha)$ such that $\mathscr{L}$ is isomorphic to $\operatorname{Hom}\left(\mathscr{L}, \hat{O}_{C_{v}}\right)$, there is exactly one $\lambda$ satisfying the preceding condition; from $(\mathscr{L}, \alpha, \lambda)$ we can reconstruct the data $(\tilde{C}, i, \tilde{e}, \pi)$. Therefore, our functor $F$ is isomorphic to the functor:

$F^{\prime}: U \sim$ sset of isomorphism classes of pairs $(\mathscr{L}, \alpha)$ on $C_{U}$ where:

(i) $\mathscr{L}$ is a coherent sheaf whose restriction on each fibre is torsion-free of rank 1 ;

(ii) $\mathscr{L}$ is isomorphic to $\underline{\operatorname{Hom}}\left(\mathscr{L}, \mathscr{O}_{\mathrm{C}_{U}}\right)$;

(iii) $\alpha$ is an isomorphism $e^{*} \mathscr{L} \stackrel{\sim}{\longrightarrow} \mathscr{O}_{U}$.

If we replace condition (ii) by a certain condition on $\operatorname{deg} \mathscr{L}$, then it follows from the work of Oda and Seshadri ([O-S]) that the functor $F^{\prime \prime}$ we obtain is representable by a "compactification of $\underline{\mathrm{Pic}}^{0}(C / T)$ " $K$. The functor $F^{\prime}$ is a closed subfunctor of $F^{\prime \prime}$, hence is representable by a closed subscheme $K_{2}$ of $K$, proper over $T$; since the fibres of $K_{2}$ over $T$ are finite, $K_{2}$ is finite over $T$. This achieves the proof of the lemma. 
Now, we consider the "Teichmuiller stacks" ${ }_{G} \mathscr{M}_{\mathrm{g}}^{0}$ and ${ }_{G} \mathscr{M}_{\mathrm{g}}([\mathrm{D}-\mathrm{M}]$ p. 106) with $G=\mathbf{Z} / 2$. Recall that ${ }_{G} \mathscr{H}_{\mathrm{g}}^{0}$ classifies smooth curves of genus $\mathrm{g}: p: C \rightarrow T$ with an element of $H^{0}\left(T, R^{1} p_{*}(\mathrm{Z} / 2)\right)$, and ${ }_{G} \mathscr{M}_{\mathrm{g}}$ is the normalization of $\mathscr{M}_{\mathrm{g}}\left[\frac{1}{2}\right]$ with respect to ${ }_{G} \mathscr{M}_{\mathrm{g}}^{0}$. Let ${ }_{G} \mathscr{C}_{\text {reg }}$ (resp. ${ }_{G} \mathscr{C}^{0}$ ) be the pull-back of $\mathscr{C}_{\text {reg }}$ over ${ }_{G} \mathscr{M}_{\mathrm{g}}$ (resp. ${ }_{G} \mathscr{M}_{\mathrm{g}}^{0}$ ). These stacks are normal, irreducible, and their fibres over $B=\operatorname{Spec}\left(Z\left[\frac{1}{2}\right]\right)$ are normal and irreducible: this follows from the same result for ${ }_{G} \mathscr{M}_{g}$ and ${ }_{G} \mathscr{M}_{g}^{0}$ (proved in [D-M]) and the fact that the morphism ${ }_{G} \mathscr{C}_{\text {reg }} \rightarrow{ }_{G} \mathscr{M}_{g}$ and ${ }_{G} \mathscr{C}^{0} \rightarrow{ }_{G} \mathscr{M}_{\mathrm{g}}^{0}$ are flat, with normal fibres and with an irreducible generic fibre. In particular, ${ }_{G} \mathscr{C}_{\text {reg }}$ is the normalization of $\mathscr{C}_{\text {reg }}$ with respect to ${ }_{G} \mathscr{C}^{0}$.

Let $\tilde{\mathscr{C}}^{0}$ be the open subset of $\tilde{\mathscr{C}}$ which classifies smooth curves. It follows from the proof of Lemma 6.5.3 that $\tilde{\mathscr{C}}^{0}$ is isomorphic to ${ }_{G} \mathscr{C}^{0}$. By the universal property of the normalization and Lemma 6.5 .3 , there is a morphism:

$$
s:{ }_{G} \mathscr{C}_{\text {reg }} \rightarrow \tilde{\mathscr{C}}_{\text {reg }}
$$

which is finite and surjective. Therefore, there is a surjective morphism ${ }_{G} \mathscr{C}_{\text {reg }} \rightarrow \overline{\mathscr{F}}$; it follows that the fibres of $\overline{\mathscr{I}}$ over $B$ are irreducible, and so are the fibres of $\mathscr{I}$. Since $p$ is surjective when $g \leqq 6$ (Lemma 6.5.2), we conclude that the fibres of $\mathscr{A}_{g-1}^{\prime}$, or equivalently the coarse moduli spaces $\mathscr{A}_{\mathrm{g}-1}$ over $k$, are irreducible for $g \leqq 6$.

Let us mention a first (and immediate) consequence of Theorems 6.5, 5.4 and 4.10.

(6.6) Proposition. Let $(A, \Theta)$ be a principally polarized abelian variety of dimension $g(2 \leqq g \leqq 5)$.

1) If $\operatorname{dim} \operatorname{Sing} \Theta=g-2,(A, \Theta)$ is a product of two principally polarized abelian varieties.

2) If $\operatorname{dim} \operatorname{Sing} \Theta=g-3,(A, \Theta)$ is a hyperelliptic Jacobian.

\section{Schottky Problem in Genus 4}

(7.1) In the moduli space $\mathscr{A}_{4}$, we look at the following subvarieties:

$N_{0}=$ locus of principally polarized abelian varieties with $\operatorname{Sing} \Theta \neq \varnothing$.

$\vec{J}_{4}=$ locus of Jacobian varieties and products of Jacobians.

$\theta_{\text {null }}=$ locus of principally polarized abelian varieties with (at least) one vanishing theta-null, i.e. such that $\operatorname{Sing} \Theta$ contains a point of order two.

It is clear that $N_{0}$ is a closed algebraic subvariety of $\mathscr{A}_{4} ; \bar{J}_{4}$ is an irreducible divisor in $\mathscr{A}_{4}([\mathrm{Ho}],[\mathrm{D}-\mathrm{M}])$. The subvariety $\theta_{\text {null }}$ is a divisor: to see this, we can find a finite covering $g: \mathscr{A}^{\prime} \rightarrow \mathscr{A}_{4}$ such that there exists on $\mathscr{A}^{\prime}$ :

- a complete family of principally polarized abelian varieties $q: \mathscr{X} \rightarrow \mathscr{A}^{\prime}$, corresponding to $\mathrm{g}$;

- a divisor $\Psi$ in $\mathscr{X}$, flat over $\mathscr{A}^{\prime}$, such that $\Psi_{t}$ is a symmetric $\Theta$ divisor on $\mathscr{X}_{t}$ for any $t$ in $\mathscr{A}^{\prime}$;

- a set of sections of $q\left(e_{\sigma}\right)_{\sigma \in \Sigma}$ such that for any $t$ in $\mathscr{A}^{\prime},\left\{e_{\sigma}(t)\right\}_{\sigma \in \Sigma}$ is the set of points of order two in $\mathscr{X}_{t}$. 
By [M 5] (or, over C, by the classical theory of theta functions), the subset: $\Sigma^{+}=\left\{\sigma \in \Sigma, e_{\sigma}\left(\mathscr{A}^{\prime}\right) \notin \Psi\right\}$ is non empty; therefore $\theta_{\text {null }}=g\left(\bigcup_{\sigma \in \Sigma^{+}} e_{\sigma}^{-1}(\Psi)\right)$ is a divisor
in $\mathscr{A}_{4}$. (7.2) Theorem. $N_{0}=\bar{J} \cup \theta_{\text {null }}$.

Proof. Let $(A, \Theta)$ be a principally polarized abelian variety with $\operatorname{Sing} \Theta \neq \emptyset$, which is neither a Jacobian nor a product; we must prove that $(A, \Theta)$ has a vanishing theta-null. By Theorems 6.5, 5.4 and $4.10,(A, \Theta)$ is isomorphic to a Prym variety satisfying condition d), f), g) or h) in Theorem 4.10.

We will prove that the line bundles $L$ which give singular points on $\Xi$ are theta-characteristic $\left(L^{2} \cong \omega_{\tilde{c}}\right)$. This is obvious in case d). In the other cases, we start from a curve $N$ which is:

- a hyperelliptic curve of genus 3 in case f);

- a genus 4 curve with one vanishing theta-null in case $\mathrm{g}$ );

- the disjoint union of two elliptic curves in case h).

In any case there is a line bundle $H$ on $N$ such that $H^{\otimes 2} \cong \omega_{N}, h^{0}(H)=2$. $C$ is obtained from $N$ by identifying points $p_{1}$ to $p_{2}, p_{3}$ to $p_{4}$, etc... We get a diagram:

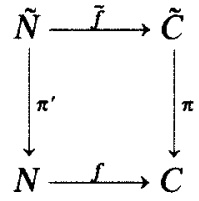

where $\pi^{\prime}$ is a two-sheeted covering ramified at $p_{1}, p_{2}, \ldots$

We put

$\left(\pi^{\prime}\right)^{-1}\left(p_{i}\right)=\left\{\tilde{p}_{i}\right\}$

The proof of Theorem 4.10 shows that the possible singularities of $\Xi$ arise from line bundles $L$ on $C$ such that:

$$
\tilde{f}^{*} L=\pi^{*} H\left(\sum \tilde{p}_{i}\right)
$$

The ramified covering $\pi^{\prime}$ defines a line bundle $\eta$ on $N$ with:

$$
\eta^{\otimes 2}=\mathcal{O}_{N}\left(\sum p_{i}\right) \quad \pi^{\prime *} \eta=\mathcal{O}_{\tilde{N}}\left(\sum \tilde{p}_{i}\right)
$$

hence

$$
f^{*} L=\pi^{*}(H \otimes \eta)
$$

Choose $M$ on $C$ such that $f^{*} M=H \otimes \eta$ and $M^{\otimes 2}=\omega_{c}$; then: $f^{*} L=\pi^{* *} f^{*} M$ $=f^{*} \pi^{*} M$ and $N m\left(\pi^{*} M\right)=\omega_{c}$.

Thus after suitable modification of $M$ we get $L=\pi^{*} M$, hence $L^{\otimes 2} \cong \omega_{\tilde{c}}$.

Using Proposition 3.4, we see that exactly half of the line bundles $M$ on $C$ with $f^{*} M=H \otimes \eta, M^{\otimes 2}=\omega_{c}$ are such that $\pi^{*} M$ belongs to $P$ (hence to Sing $\Xi$ ). Thus we find:

2 singular points of $\Xi$ in case $f$ ); 
1 singular point in case $\mathrm{g}$ );

3 singular points in case $h$ ).

All these points correspond to vanishing theta-nulls.

(7.3) Proposition. The divisor $\theta_{\text {null }}$ is the closure of the locus of Prym varieties associated to a non-singular curve $C$ of genus five with one vanishing theta-null $N$ and $h^{0}\left(\tilde{C}, \pi^{*} N\right)$ even.

Proof. We have to prove that cases f), g), h) are specializations of case d). We keep the notation used in Theorem 7.2.

(7.3.1) In the canonical embedding (defined by $\omega_{c}$ ), $C$ is a complete intersection of 3 quadrics, except in case g) if $h^{0}\left(H\left(-p_{1}-p_{2}\right)\right) \geqq 1$. This is seen as for nonsingular curves: $C$ is contained in 3 linearly independant quadrics (by RiemannRoch); if the intersection of these quadrics is bigger than $C$, it must be a cubic scroll, and $C$ must be trigonal - thus the only possibility is the exceptional case mentioned above. This case is a specialization of the generic case g), so we may always assume that $C$ is a complete intersection of 3 quadrics.

(7.3.2) Furthermore $C$ is contained in a quadric $Q$ of rank $\leqq 3$. This is because of the diagram:

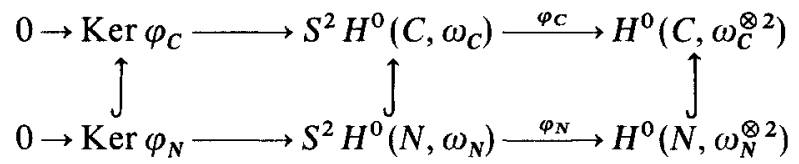

which implies that $\operatorname{Ker} \varphi_{C}$ contains a quadratic relation of rank 3 in case f) and $g$ ), and of rank 2 in case $h$ ).

(7.3.3) In cases $f$ ) and g) we fix the quadric $Q$ and a two-dimensional linear subspace $\Pi \subset Q$, and deform the other quadrics; in case h), we let $\Pi$ be the singular locus of $Q$ and deform $Q$ in a rank 3 quadric containing $\Pi$. Thus we get a family of curves in $\mathbf{P}^{4}$ :

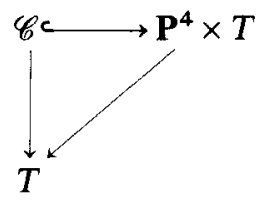

such that $\mathscr{C}_{0}=C$, and $\mathscr{C}_{t}$ is non-singular for $t \neq 0$.

Let $\mathscr{I}=$ ideal of $(\Pi \times T) \cap \mathscr{C}$ in $\mathscr{C}, \mathscr{L}=\operatorname{Hom}_{\mathscr{O} \mathscr{C}}\left(\mathscr{I}, \mathcal{O}_{\mathscr{C}}\right)$. It is easy to check that $\mathscr{L}_{\mid C} \cong f_{*}(H)$, while $\mathscr{L}_{\mid \mho_{t}}$ is a "vanishing theta-null".

Thus we have found a family of curves $\mathscr{C} \rightarrow T$ and a coherent sheaf $\mathscr{L}$ on $\mathscr{C}$ such that:

- For $t \neq 0, \mathscr{C}_{t}$ is a non-singular genus 5 curve, $\mathscr{L}_{t}$ is a line bundle with $\mathscr{L}_{t}^{\otimes 2}=\omega_{\mathscr{C}_{t}}$ and $h^{0}\left(\mathscr{L}_{t}\right)=2$;

$-\mathscr{C}_{0}=C$ and $\mathscr{L}_{0} \cong f_{*}(H)$. 
Moreover, from the multiplication $\mathscr{I} \otimes \mathscr{I} \rightarrow \omega_{\mathscr{G} / T}^{-1} \subset \mathcal{O}_{\mathscr{C}}$ we get a duality $\mathscr{L} \otimes \mathscr{L}$ $\rightarrow \omega_{\mathscr{G} / T}$.

(7.3.4) There exists a line bundle $L_{0}$ on $C$ such that:

$$
L_{0}^{\otimes 2} \cong \omega_{C}, \quad f^{*} L_{0}=H \otimes \eta, \quad h^{0}\left(L_{0}\right) \text { even }
$$

(see Theorem 7.2). The variety of line bundles $M$ on $\mathscr{C}_{t}$ with $M^{\otimes 2} \cong \omega_{\mathscr{C}_{t}}$ is étale over $T$, hence by passing to an étale covering of $T$, we may extend $L_{0}$ to a line bundle $L$ on $\mathscr{C}$ with $L^{\otimes 2} \cong \omega_{\mathscr{C} / T}$. Then by Theorem $1.1, h^{0}\left(\mathscr{C}_{t}, L_{t}\right)$ is even for any $t$ in $T$.

(7.3.5) Define $\tilde{\mathscr{C}}=\operatorname{Spec}\left(\mathcal{O}_{\mathscr{G}} \oplus\left(\mathscr{L} \otimes L^{-1}\right)\right)$, where the algebra structure is given by the morphism:

$$
\left(\mathscr{L} \otimes L^{-1}\right) \otimes\left(\mathscr{L} \otimes L^{-1}\right) \rightarrow \mathscr{O}_{\mathscr{C}}
$$

deduced from the duality $\mathscr{L} \otimes \mathscr{L} \rightarrow \omega_{\mathscr{G} / \mathrm{T}}$. Then:

- for $t \neq 0, \pi_{t}: \tilde{\mathscr{C}}_{t} \rightarrow \mathscr{C}_{t}$ is an unramified two-sheeted covering, and $h^{0}\left(\pi_{t}^{*} \mathscr{L}_{t}\right)$ $=h^{0}\left(\mathscr{L}_{t}\right)+h^{0}\left(L_{t}\right)$ is even;

$-\pi_{0}: \tilde{\mathscr{C}}_{0} \rightarrow \mathscr{C}_{0}$ can be identified to the morphism $\pi: \tilde{C} \rightarrow C$.

This proves that situations $f, g), h$ ) are specializations of situation $d$ ).

(7.3.6) One sees easily that a morphism $\tilde{C} \rightarrow C_{0}$ in case d) with $C_{0}$ singular is a specialization of an unramified two-sheeted covering $\tilde{C} \rightarrow C$ in case d) with $C$ non-singular. This achieves the proof of Proposition 7.3.

(7.4) Theorem. In characteristic zero, the divisor $\theta_{\text {null }}$ is irreducible.

(7.4.1) Proof. We can suppose $k=\mathbf{C}$.

Let $f: X \rightarrow S$ be a complete family of curves of genus 5 with one vanishing theta-null; this means that there is a line bundle $N$ on $X$ whose restriction $N_{s}$ to $X_{s}$ satisfies:

$N_{s}^{2} \cong \omega_{X_{s}} ; \quad h^{0}\left(N_{s}\right)=2 \quad$ for any $s$ in $S$.

We can take for $S$ an irreducible smooth variety (fix a rank 3 quadric $Q_{0}$ in $\mathbf{P}^{4}$; a generic curve of genus 5 with one vanishing theta-null can be obtained as the intersection of $Q_{0}$ with two arbitrary quadrics).

Let us denote by $\left(J X_{s}\right)_{2}$ the group of points of order two in $J X_{s}$, isomorphic to $H^{1}\left(X_{s}, \mathbf{Z} / 2\right)$. We consider the covering $Z$ of $S$ whose fibre over a point $s$ in $S$ is $\left(J X_{s}\right)_{2}$. The quadratic form $q$ on $\left(J X_{s}\right)_{2}$ given by:

$$
q(\eta)=h^{0}\left(X_{s}, N_{s} \otimes \eta\right) \quad(\bmod 2)
$$

splits the covering $Z$ into two parts: we denote by $Z_{0}$ the subcovering corresponding to $q=0$. We have to prove that $Z_{0}$ is irreducible; or equivalently, fixing a point $s$ in $S$, that $\Pi_{1}(S, s)$ acts transitively on the set of $\eta \in\left(J X_{s}\right)_{2}$ with $q(\eta)=0$. Observe that it is enough to prove this assertion for one family $X \rightarrow S$ (not necessarily complete) of curves of genus 5 having a vanishing theta-null. We will reduce this problem to a statement about plane curves by using the following known facts about curves of genus five. 
(7.4.2) Let $X$ be a non-singular curve of genus 5, which is neither hyperelliptic nor trigonal. Then $X$ is canonically embedded in $\mathbf{P}^{4}$ as a complete intersection of three quadrics. Hence the linear system of quadrics in $\mathbf{P}^{4}$ containing $X$ is a projective plane $\Pi$. The discriminant locus (curve of singular quadrics in $I T$ ) is a plane quintic with ordinary double points; such a double point appears if and only if the corresponding quadric is of rank 3, which means that $X$ has a vanishing theta-null. The rank 4 quadrics contain two systems of generatrices, which define a double covering $\pi: \tilde{C} \rightarrow C$; if $C$ is singular, $\pi$ is a degree two morphism with property $(*)$ of $\S 3$. In any case the Prym variety associated to $(\tilde{C}, C)$ is isomorphic to the Jacobian $J X$. Note also that $h^{0}\left(\pi^{*} \mathcal{O}_{C}(1)\right)$ is odd.

Conversely, given a stable plane quintic $C$, a degree two morphism $\pi: \tilde{C} \rightarrow C$ with property $(*)$ and such that $h^{0}\left(\pi^{*} \mathcal{O}_{C}(1)\right)$ is odd, there exists a unique curve $X$ of genus 5 such that $J X \cong \operatorname{Prym}(\tilde{C}, C)$.

Furthermore, this construction can be done locally over any base variety. Let $\mathscr{C} \rightarrow S$ be a family of stable plane quintics (with $\mathscr{C} \subset \mathbf{P}_{S}^{2}$ ) and $\pi: \tilde{\mathscr{C}} \rightarrow \mathscr{C}$ a degree 2 morphism such that for each $s$ in $S$, the induced morphism $\pi_{s}: \tilde{\mathscr{C}}_{s} \rightarrow \mathscr{C}_{s}$ has property $(*)$ and $h^{0}\left(\pi_{s}^{*} \mathcal{O}_{\mathscr{C}_{s}}(1)\right)$ is odd. Then locally over $S$, there exists a family $X \rightarrow S$ of non-singular curves of genus 5 such that $J X_{s} \cong \operatorname{Prym}\left(\tilde{\mathscr{C}}_{s}, \mathscr{C}_{s}\right)$ for each $s$.

In the sequel of the proof, we are going to translate the assertion about the action of $\Pi_{1}(S)$ on $\left(J X_{s}\right)_{2}$ as an assertion about $\mathscr{C}$ and $\tilde{\mathscr{C}}$, and then we shall prove by monodromy methods that this last assertion is true for a good choice of the family $\mathscr{C} \rightarrow S$.

(7.4.3) Now let $g: C^{\prime} \rightarrow T$ be a family of plane quintics with exactly one ordinary double point. We denote by $R_{t}(t \in T)$ the set of isomorphism classes of degree 2 morphisms $\pi: \tilde{C}^{\prime} \rightarrow C_{t}^{\prime}$ with property $(*)$ and such that $h^{0}\left(\pi^{*} \mathscr{O}_{C_{t}}(1)\right)$ is odd. Let $p: S \rightarrow T$ be the covering of $T$ such that $p^{-1}(t)=R_{t}$; we put $C=C^{\prime} \times_{T} S$. We get a degree 2 morphism over $S$ :

$$
\pi: \tilde{C} \rightarrow C \text {. }
$$

To this family of plane quintics with covering we associate (7.4.2) a family $X \rightarrow S$ of non-singular curves of genus 5 having one vanishing theta-null. Recall that there is a canonical isomorphism:

$$
J X_{s} \stackrel{\sim}{\longrightarrow} \operatorname{Prym}\left(\tilde{C}_{s}, C_{s}\right) .
$$

Let $N_{s}$ be the normalization of $C_{s}$. By [M2, p. 332], there is an isomorphism:

$$
\left(J X_{s}\right)_{2} \stackrel{\sim}{\longrightarrow} H^{1}\left(N_{s}, \mathbf{Z} / 2\right)
$$

which is equivariant with respect to the action of $\pi_{1}(S, s)$.

To the quadratic form $q$ on $\left(J X_{s}\right)_{2}$ corresponds a form $q^{\prime}$ on $H^{1}\left(N_{s}, \mathbf{Z} / 2\right)$, invariant under the action of $\Pi_{1}(S, s)$. We wish to prove that for a certain choice of the family $C^{\prime} \rightarrow T$, the group $\Pi_{1}(S, s)$ acts transitively on the set of $\eta \in H^{1}\left(N_{s}, \mathbf{Z} / 2\right)$ with $q^{\prime}(\eta)=0, \eta \neq 0$.

Put $t=p(s)$. The group $\Pi_{1}(T, t)$ acts on $R_{t}$ and $\Pi_{1}(S, s)$ is isomorphic to the isotropy subgroup of $s \in R_{t}$.

A covering $\pi: \tilde{C} \rightarrow C_{s}$ with property (*) is given by a cycle in $H_{1}\left(C_{s}, \mathbf{Z} / 2\right)$ 
which does not belong to $\operatorname{Im} H_{1}\left(N_{s}, \mathbf{Z} / 2\right)$; or in terms of cohomology, by a linear form:

$$
\varphi: H^{1}\left(C_{s}, \mathbf{Z} / 2\right) \rightarrow \mathbf{Z} / 2
$$

such that

$$
\varphi(\omega)=1,
$$

where $\omega$ denotes the only non-zero element of the kernel of the cup product on $H^{1}\left(C_{s}, \mathbf{Z} / 2\right)$. The composition:

$$
\operatorname{Ker} \varphi \hookrightarrow H^{1}\left(C_{s}, \mathbf{Z} / 2\right) \rightarrow H^{1}\left(N_{s}, Z / 2\right)
$$

is an isomorphism respecting the symplectic structure and the action of $\Pi_{1}(S, s)$.

We now wish to express the condition that the degree 2 morphism $\pi: \tilde{C} \rightarrow C_{s}$ corresponding to $\varphi$ belongs to $R_{t}$, that is $h^{0}\left(\pi^{*} \mathcal{O}_{\mathscr{C}_{s}}(1)\right)$ is odd. We can embed the family $C \rightarrow S$ in a larger family of plane quintics $h: D \rightarrow U$ such that the fibres of $h$ over $U-S$ are non-singular. Let us choose a path in $U$ from $s$ to a point $u$ in $U-S$. By Lefschetz theory we get an injective map:

$$
H^{1}\left(C_{s}, \mathrm{Z} / 2\right) \hookrightarrow H^{1}\left(D_{u}, \mathrm{Z} / 2\right)
$$

which identifies $H^{1}\left(C_{s}, Z / 2\right)$ to the orthogonal of the "vanishing cycle" $\omega$. The form $\varphi$ determines an element $\gamma \in H^{1}\left(D_{u}, \mathbf{Z} / 2\right)$ such that:

$$
\varphi(x)=(x \cdot \gamma) \text { for } x \in H^{1}\left(C_{s}, Z / 2\right) .
$$

There is a unique deformation invariant quadratic form $r$ on $H^{1}\left(D_{u}, \mathbf{Z} / 2\right)$ whose associated bilinear form is the cup-product (see [M 1]); algebraically, it is given by:

$$
r(\eta)=h^{0}\left(D_{u}, \mathcal{O}_{D_{u}}(1) \otimes \eta\right)+1 \quad \text { for } \eta \in\left(J D_{u}\right)_{2} \cong H^{1}\left(D_{u}, \mathbf{Z} / 2\right)
$$

and its Arf invariant is equal to 1 .

The condition $\varphi \in R_{t}$ is equivalent to $r(\gamma)=1$. Notice that $\operatorname{Ker}(\varphi)$ is the subspace of $H^{1}\left(D_{u}, Z / 2\right)$ orthogonal to the hyperbolic plane generated by $\omega$ and $\gamma$. Also, since $\omega$ is a vanishing cycle and $r$ is deformation invariant, one must have $r(\omega)=1$. It follows that the condition $q(\gamma)=1$ is equivalent to the following:

\section{(A1) The Arf Invariant of the Restriction of $r$ to $\operatorname{Ker}(\varphi)$ is 0}

Note that this is consistent with the fact that the Arf invariant of the form $q$ (7.4.1) is 0 .

(7.4.4) We are now reduced to a monodromy computation. We will use the method of $\left[\mathrm{A}^{\prime} \mathrm{C}\right]$. We consider the plane singularity:

$$
x^{5}+x y^{3}=0 \text {. }
$$

A versal deformation of this singularity is given by: 


$$
\begin{aligned}
& x^{5}+x y^{3}+t_{1} x^{4} y+t_{2} x^{4}+t_{3} x^{3} y+t_{4} x^{3}+t_{5} x^{2} y+t_{6} x^{2}+t_{7} x y \\
& +t_{8} y^{2}+t_{9} x+t_{10} y+t_{11}=0 .
\end{aligned}
$$

Note that the projective completion of this curve has always an ordinary double point at infinity.

Recall the notation of $\left[\mathrm{A}^{\prime} \mathrm{C}\right]$ :

$\mathscr{D}_{n}=$ a small ball in $\mathbf{C}^{11}$,

$\Delta \subset \mathscr{D}_{\eta}=$ discriminant locus,

$B_{\varepsilon}=$ a small ball in $\mathbf{C}^{2}$.

We take $T=\mathscr{D}_{\eta}-\Delta$, the family $C^{\prime} \rightarrow T$ being given by the restriction of the versal deformation.

Fix $t \in T$; put $C_{t}^{\prime}=C, F=C \cap B_{\varepsilon}$. A'Campo's method allows to compute the local monodromy, that is the action of $\Pi_{1}(T, t)$ on $H_{1}(F, \mathbf{Z})$, or equivalently on $H_{c}^{1}(F, Z)$. But now there is an equivariant exact sequence:

$$
0 \rightarrow H^{0}(C, \mathbf{Z}) \rightarrow H^{0}(C-F, \mathbf{Z}) \rightarrow H_{c}^{1}(F, Z) \rightarrow H^{1}(C, \mathbf{Z}) .
$$

Since $C-F$ is connected, and $\operatorname{dim} H_{c}^{1}(F)=\operatorname{dim} H^{1}(C)=11$, we get an isomorphism:

$$
H_{\mathrm{c}}^{1}(F, \mathrm{Z} / 2) \stackrel{\sim}{\longrightarrow} H^{1}(C, \mathrm{Z} / 2)
$$

which respects the symplectic structure and the action of $\Pi_{1}(T)$.

We are going to choose a linear form $\varphi$ on $H^{1}(C, Z / 2)$ as in (7.4.3), and prove that the subgroup of $\Pi_{1}(T, t)$ which leaves $\varphi$ invariant acts transitively on the set of $\eta \in \operatorname{Ker} \varphi$ with $q(\eta)=0, \eta \neq 0$; by what we have seen, this will prove the Theorem. Now we apply A'Campo's method. To get a "confluence de Morse", we use the following succession of blowing up, deformation and blowing down:
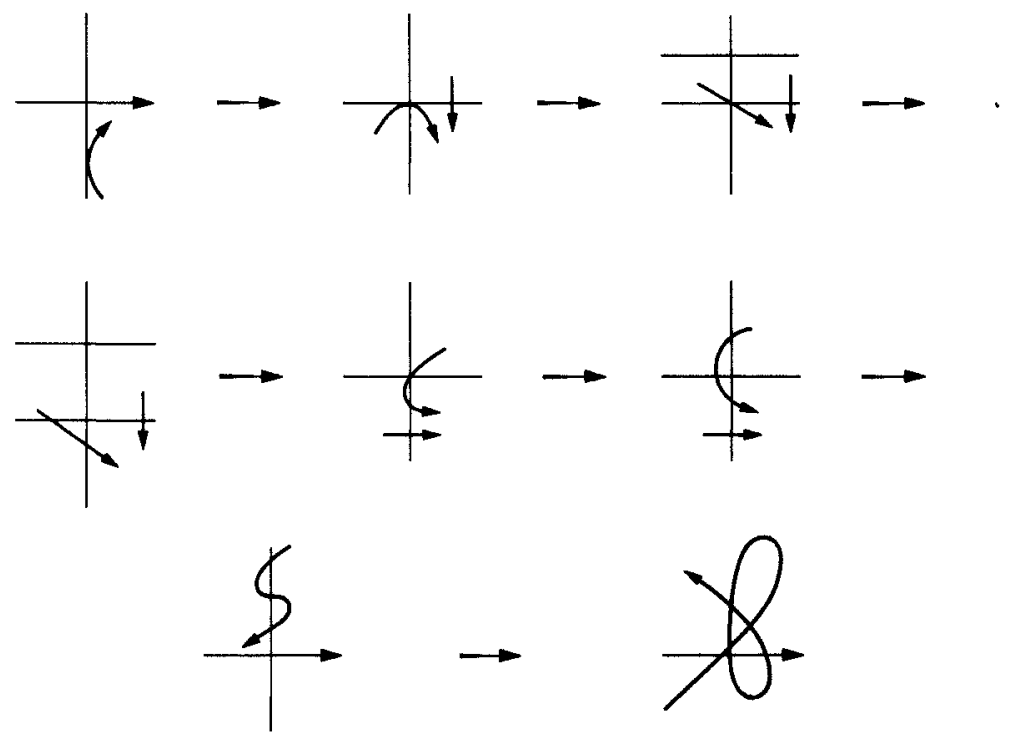
One checks immediately that these operations can be done without altering the double point at infinity.

We obtain finally the following "partage":

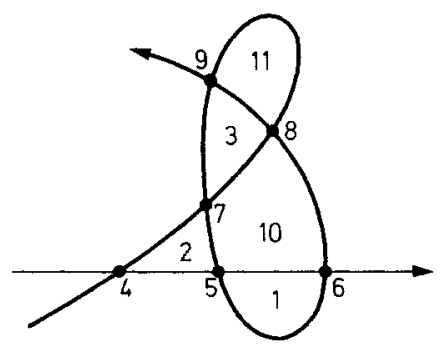

which gives the Dynkin diagram:

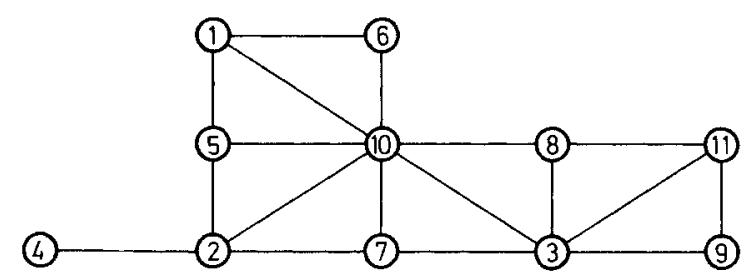

Recall that this means that there is a basis $\delta_{1}, \ldots, \delta_{11}$ of $H^{1}(C, \mathbf{Z} / 2)$ with:

$$
\begin{aligned}
\left(\delta_{i} \cdot \delta_{j}\right) & =1 & & \text { if }(i) \text { and }(i) \text { are connected by a line in the diagram }(i \neq j) ; \\
& =0 & & \text { if they are not. }
\end{aligned}
$$

With the notation of (7.4.3), we have:

$$
\omega=\delta_{4}+\delta_{5}+\delta_{6} \text {. }
$$

The quadratic form $r$ is characterized by $r\left(\delta_{i}\right)=1$ for all $i$.

We choose the linear form $\varphi$ on $H^{1}(C, \mathbf{Z} / 2)$ defined by:

$$
\varphi\left(\delta_{6}\right)=1, \quad \varphi\left(\delta_{i}\right)=0 \quad \text { for } i \neq 6 .
$$

We must check condition $(A I)$. It is convenient to use a symplectic basis for $\operatorname{Ker}(\varphi)$; we take:

$$
\begin{array}{ll}
\alpha_{1}=\delta_{4} ; & \beta_{1}=\delta_{2}, \\
\alpha_{2}=\delta_{9} ; & \beta_{2}=\delta_{11}, \\
\alpha_{3}=\delta_{1} ; & \beta_{3}=\delta_{4}+\delta_{5}, \\
\alpha_{4}=\delta_{4}+\delta_{7} ; & \beta_{4}=\delta_{3}+\delta_{9}+\delta_{11}, \\
\alpha_{5}=\delta_{8}+\delta_{9} ; & \beta_{5}=\delta_{1}+\delta_{3}+\delta_{4}+\delta_{5}+\delta_{7}+\delta_{8}+\delta_{10}+\delta_{11} .
\end{array}
$$


Then:

$$
\begin{aligned}
& \left(\alpha_{i} \cdot \alpha_{j}\right)=0, \quad\left(\beta_{i} \cdot \beta_{j}\right)=0, \quad\left(\alpha_{i} \cdot \beta_{j}\right)=0, \quad \text { if } i \neq j, \\
& \left(\alpha_{i} \cdot \beta_{i}\right)=1 .
\end{aligned}
$$

One checks immediately that:

$$
\sum_{i=1}^{5} r\left(\alpha_{i}\right) \cdot r\left(\beta_{i}\right)=0
$$

i.e. condition $(A I)$ is verified.

The monodromy group is generated by the transvections $T_{i}$ :

$$
T_{i}(x)=x+\left(x \cdot \delta_{i}\right) \delta_{i}, \quad x \in H^{1}(C, \mathbf{Z} / 2) .
$$

We will study the orbits in $\operatorname{Ker}(\varphi)$ of the group $G$ generated by the transvections $T_{i}$ which leave $\varphi$ invariant, that is the $T_{i}$ 's for $i \neq 6$. We set up sume notation:

If $x=\sum p_{i} \alpha_{i}+\sum q_{j} \beta_{j}$, we note:

$$
x=\left[\begin{array}{lll}
p_{1} & \ldots & p_{5} \\
q_{1} & \ldots & q_{5}
\end{array}\right] \text {. }
$$

We write $x \equiv y$ if $y=g \cdot x$ for some $g$ in $G$.

The action of $T_{4}, T_{2}, T_{9} . T_{11}, T_{1}$ is very simple; for instance:

$$
T_{4}(x)=\left[\begin{array}{cc}
p_{1}+q_{1} & \cdots \\
q_{1} & \cdots
\end{array}\right]
$$

(which means that only the coordinate $p_{1}$ is modified).

Then we have:

$$
\begin{aligned}
& T_{5}(x)=\left[\begin{array}{ccc}
p_{1}+\left(q_{1}+p_{3}\right) & \cdot & \cdot \\
\cdot & \cdot q_{3}+\left(q_{1}+p_{3}\right) & \cdot
\end{array}\right], \\
& T_{7}(x)=\left[\begin{array}{ccc}
p_{1}+\left(q_{1}+q_{4}\right) & . & \cdot p_{4}+\left(q_{1}+q_{4}\right) \\
. & . & .
\end{array}\right] \text {. }
\end{aligned}
$$

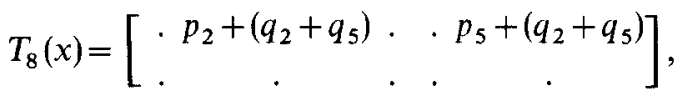

$$
\begin{aligned}
& T_{3}(x)=\left[\begin{array}{ll}
\cdot p_{2}+\left(p_{4}+p_{2}+q_{2}\right) & \cdot \\
\cdot q_{2}+\left(p_{4}+p_{2}+q_{2}\right) & \cdot q_{4}+\left(p_{4}+p_{2}+q_{2}\right)
\end{array}\right] \text {, } \\
& T_{10}(x)=\left[\begin{array}{ccccc}
p_{1}+r & p_{2} & p_{3}+r & p_{4}+r & p_{5}+r \\
q_{1} & q_{2} & q_{3}+r & q_{4}+r & q_{5}+r
\end{array}\right]
\end{aligned}
$$

with $r=q_{1}+p_{3}+q_{3}+p_{4}+q_{4}+p_{5}+q_{5}$.

a) There exists in $G \cdot x$ an element with $p_{1}=q_{1}=0$.

Proof. If $x$ does not have this property, we can suppose (using $T_{4}, T_{2}$ ) that: 
$x=\left[\begin{array}{lllll}1 & . & . & . & \ddots \\ 0 & . & . & . & .\end{array}\right]$

Using $T_{7}$ and $T_{4}, T_{2}$, we can always suppose $p_{4}=1$. Then using $T_{3}$ and $T_{9}, T_{11}$ we can suppose $q_{4}=1$. Then we apply $T_{7}$.

b) There exists in $G \cdot x$ an element with $p_{1}=q_{1}=p_{2}=q_{2}=0$.

Proof. Using a) and $T_{9}, T_{11}$, we can suppose:

$x=\left[\begin{array}{lllll}0 & 1 & \cdot & \cdot & \cdot \\ 0 & 0 & . & . & .\end{array}\right]$.

If $q_{5}=1$, we apply $T_{8}$.

If $q_{5}=0, q_{4}=0$, note that using eventually $T_{8}, T_{11}$ we can suppose $q_{1}+p_{3}+q_{3}+p_{4}+q_{4}+p_{5}+q_{5}=1$. Then, we apply $T_{7} T_{8} T_{10}$.

If $q_{5}=0, q_{4}=1$, we manage in the same way to obtain $q_{1}+p_{3}+q_{3}+p_{4}+q_{4}$ $+p_{5}+q_{5}=0$. Then we apply $T_{8} T_{10} T_{7}$.

c) There exists in $G \cdot x$ an element with $p_{i}=q_{i}=0 \quad(i=1,2,3)$.

Proof. Applying $T_{1}$ we can suppose:

either $x=\left[\begin{array}{lllll}0 & 0 & 1 & . & . \\ 0 & 0 & 1 & . & .\end{array}\right]$ or $x=\left[\begin{array}{lllll}0 & 0 & 1 & . & . \\ 0 & 0 & 0 & . & .\end{array}\right]$.

We put $r=p_{4}+q_{4}+p_{5}+q_{5}$.

$\left.c_{1}\right) \quad x=\left[\begin{array}{lllll}0 & 0 & 1 & . & . \\ 0 & 0 & 1 & . & .\end{array}\right]$.

If $r=1$, we apply $T_{10} T_{1} T_{2} T_{4} T_{10} T_{2} T_{5}$.

If $r=0$, using possibly $T_{1} T_{10} T_{5}$ we can suppose $q_{4}=1$. Then we apply $T_{10} T_{7}$.

$\left.c_{2}\right) \quad x=\left[\begin{array}{lllll}0 & 0 & 1 & \cdot & \cdot \\ 0 & 0 & 0 & . & .\end{array}\right]$.

If $r=1$, we apply $T_{10} T_{5}$.

If $r=0$, using $T_{5} T_{1} T_{10}$ we can suppose $q_{4}=1$. Then applying $T_{5} T_{7}$ we find again situation $\mathrm{c}_{1}$ ).

d) Let us now write:

$\left[\begin{array}{lllll}0 & 0 & 0 & p_{4} & p_{5} \\ 0 & 0 & 0 & q_{4} & q_{5}\end{array}\right]=\left(\begin{array}{ll}p_{4} & p_{5} \\ q_{4} & q_{5}\end{array}\right)$

Then we have:

$\left(\begin{array}{cc}1 & p_{5} \\ q_{4} & 0\end{array}\right) \equiv\left(\begin{array}{cc}1 & p_{5}+1 \\ q_{4} & 0\end{array}\right) \quad$ by $T_{3} T_{8} T_{9} T_{3}$ 


$$
\begin{aligned}
\left(\begin{array}{cc}
1 & p_{5} \\
q_{4} & 0
\end{array}\right) & \equiv\left(\begin{array}{cc}
1 & p_{5}+1 \\
q_{4}+1 & 1
\end{array}\right) & & \text { by } T_{3} T_{11} T_{8}, \\
\left(\begin{array}{cc}
0 & p_{5} \\
q_{4} & 1
\end{array}\right) & \equiv\left(\begin{array}{cc}
0 & p_{5} \\
q_{4}+1 & 1
\end{array}\right) & & \text { by } T_{8} T_{11} T_{9} T_{3} T_{8}, \\
\left(\begin{array}{cc}
1 & p_{5} \\
0 & q_{5}
\end{array}\right) & \equiv\left(\begin{array}{cc}
0 & p_{5} \\
1 & q_{5}
\end{array}\right) & & \text { by } T_{7} T_{3} T_{2} T_{7} T_{4} T_{2} T_{11} T_{3} T_{7} T_{9} T_{3}, \\
\left(\begin{array}{cc}
p_{4} & p_{5} \\
0 & q_{5}
\end{array}\right) & \equiv\left(\begin{array}{cc}
p_{4} & p_{5}+1 \\
1 & q_{5}+1
\end{array}\right) & & \text { if } p_{4}+p_{5}+q_{5}=1, \text { by } T_{7} T_{2} T_{4} T_{5} T_{1} T_{2} T_{10} .
\end{aligned}
$$

From this, it is immediate to conclude that the non-zero elements of $H^{1}\left(N_{s}, Z / 2\right)$ fall into two orbits under $G$, according to the value of the quadratic form $r$. This achieves the proof of Theorem 7.4.

Let us mention the following corollary of Theorem 7.4 :

(7.5) Proposition. Assume char $(k)=0$. For $(A, \Theta)$ generic in $\theta_{\text {null }}$, the divisor $\Theta$ has only one ordinary double point (corresponding to the vanishing theta-null).

Proof. It is clearly enough to prove the result for one principally polarized abelian variety $(A, \Theta)$ in $\theta_{\text {null }}$. Let $C$ be a non-singular curve of genus 5 , not trigonal, having precisely two vanishing theta-nulls; i.e. two line bundles, $H$ and $H^{\prime}$, with:

$$
H^{\otimes 2} \cong H^{\prime \otimes 2} \cong \omega_{C} \quad \text { and } \quad h^{0}(H)=h^{0}\left(H^{\prime}\right)=2 .
$$

Then $\eta=H^{\prime} \otimes H^{-1}$ satisfies $\eta^{\otimes 2}=\mathcal{O}_{C}$, thus determines a two-sheeted covering $\pi: \tilde{C} \rightarrow C$. The only singularity in the divisor $\Xi$ of the associated Prym variety $(P, \Xi)$ corresponds to the line bundle $\pi^{*} H$; we wish to compute the tangent cone to $\Xi$ at this singular point. According to [M2, p. 343], we must take a basis $s_{1} \ldots s_{4}$ of $H^{0}\left(\tilde{C}, \pi^{*} H\right)$; if we identify the tangent space to $P$ to $H^{0}\left(C, \omega_{c} \otimes \eta\right)^{*}$, the equation of the tangent cone is given by the Pfaffian of the matrix:

$$
M=\left(s_{i} \otimes \imath^{*} s_{j}-s_{j} \otimes l^{*} s_{i}\right)_{1 \leqq i, j \leqq 4} .
$$

Using the decomposition:

$$
H^{0}\left(\tilde{C}, \pi^{*} H\right)=H^{0}(C, H) \oplus H^{0}(C, H \otimes \eta)
$$

we get a basis $s_{1}, s_{2}, t_{1}, t_{2}$ of $H^{0}\left(\tilde{C}, \pi^{*} H\right)$ with:

$$
l^{*} s_{i}=s_{i} \quad l^{*} t_{j}=-t_{j} .
$$

Then, the above matrix becomes:

$$
M=2\left(\begin{array}{c:c}
0 & \left(a_{i j}\right) \\
\hdashline-\left(a_{i j}\right) & 0
\end{array}\right)
$$

with $a_{i j}=s_{i} \otimes t_{j} \in H^{0}\left(C, \omega_{c} \otimes \eta\right) \quad(1 \leqq i, j \leqq 2)$.

Thus, the tangent cone is given by the equation:

$$
a_{11} a_{22}-a_{12} a_{21}=0 \text {. }
$$


Now from the exact sequence:

$$
0 \rightarrow H^{-1} \stackrel{\left(s_{2},-s_{1}\right)}{\longrightarrow} \mathcal{O}_{\mathrm{C}}^{2} \stackrel{\left(s_{1}, s_{2}\right)}{\longrightarrow} H \rightarrow 0
$$

we deduce after tensorization with $H \otimes \eta$ that the natural map:

$$
H^{0}(C, H) \otimes H^{0}(C, H \otimes \eta) \rightarrow H^{0}\left(C, \omega_{C} \otimes \eta\right)
$$

is injective. Hence the $\left(a_{i j}\right)$ 's are linearly independant in $H^{0}\left(C, \omega_{C} \otimes \eta\right)$. Therefore the tangent cone to $\Xi$ at the singular point is of rank 4 , which means that this point is an ordinary double point.

(7.6) Note that this is in contrast with the situation for Jacobians: the $\Theta$-divisor of a Jacobian variety has two ordinary double points, which can collapse in one non-ordinary double point.

(7.7) Remark. Let us denote by $p$ the projection from the Siegel upper halfspace $H_{4}$ onto $\mathscr{A}_{4}$. Theorem 7.2 and the work of Androtti-Mayer ([A-M]) give a theoretical way to write an equation for the divisor $p^{-1}\left(\bar{J}_{4}\right)$ in terms of thetanulls. Notice first that the procedure given in $[\mathrm{A}-\mathrm{M}]$ can be slightly modified to get only one equation for $p^{-1}\left(N_{0}\right)$. Namely the fact that a point $z$ in $H_{4}$ belongs to $p^{-1}\left(N_{0}\right)$ is equivalent to the following (here we use freely the notation and results of $[\mathrm{A}-\mathrm{M}]$, p. 227]):

the linear space of codimension 5 in $\mathbf{P}^{15}$ given by:

$$
\sum_{\mu} \lambda_{\mu} \cdot \theta_{2}[\mu](0, z)=0
$$

(L) $\sum_{\mu} \lambda_{\mu} \cdot \frac{\partial^{2} \theta_{2}[\mu]}{\partial u_{\alpha}^{2}}(0, z)=0 \quad \alpha=1, \ldots, 4$

has a non-empty intersection with the Kummer-Wirtinger variety $K(z) \subset \mathbf{P}^{15}$.

This gives one condition on $z$; it is expressed by the vanishing of a function $F(z)$ which is a homogeneous polynomial with rational coefficients in the "theta-nulls" $C(r, \mu, z)$. Note that $F(z)$ is the Chow form of $K(z)$ applied to the Pluicker coordinates of the linear system $(L)$.

We don't know whether the divisor given by $\theta(0, z)=0$ in $H_{4}$ is irreducible; however we know that each of its components has multiplicity 1 : this follows from the "heat equations" ([A-M]) and Theorem 7.4. Let us denote by $\varphi(z)$ the product of all the theta-null functions corresponding to even characteristics; in classical notation:

$$
\varphi(z)=\prod_{\varepsilon, \varepsilon^{\prime}=0} \theta\left[\begin{array}{l}
\varepsilon \\
\varepsilon^{\prime}
\end{array}\right](0, z) .
$$

Then it follows from what we have seen that a certain power of $\varphi(z)$, say $\varphi(z)^{s}$, divides $F(z)$, and the holomorphic function:

$$
\varphi(z)^{-s} \cdot F(z)
$$

gives an equation for $p^{-1}\left(\vec{J}_{4}\right)$. 


\section{Schottky Problem in Genus 5}

In genus 5, Theorem 4.10 (together with Theorem 6.5) gives a complete description of the locus $N_{1}$ of principally polarized abelian varieties with $\operatorname{dim} \operatorname{Sing} \Theta \geqq 1$. $N_{1}$ has already many components. However, if we still denote by $\theta_{\text {null }}$ the divisor in $\mathscr{A}_{5}$ of principally polarized abelian varieties with (at least) one vanishing thetanull, we have:

(8.1) Proposition. $\bar{J}_{5}$ is the only component of $N_{1}$ not contained in $\theta_{\text {null }}$.

Proof. It is well-known that a generic Jacobian has no vanishing theta-null ([F]). Clearly any product of principally polarized abelian varieties belongs to $\theta_{\text {null }}$. Thus we must prove that every Prym variety in case c), f), h), i) or j) of Theorem 4.10 has a vanishing theta-null.

(8.1.1) Case $c$. We may assume that $C$ is non-singular, since this is the generic case and $\theta_{\text {null }}$ is closed.

Let $E$ be an elliptic curve, $h: C \rightarrow E$ a two-sheeted covering ramified at the points $\tilde{r}_{1}, \ldots, \tilde{r}_{10}$ of $C, \sigma: C \rightarrow C$ the corresponding involution; to this covering is associated a line bundle $\delta$ such that:

$$
\delta^{2} \cong \mathcal{O}_{E}\left(\sum r_{i}\right) \text { with } \quad r_{i}=h\left(\tilde{r}_{i}\right)
$$

Let $\pi: \tilde{C} \rightarrow C$ be an unramified two-sheeted covering, given by a line bundle $\eta$ on $C$ with $\eta^{2}=\mathcal{O}_{C}$; we consider the Prym variety $(P, \Xi)$ associated to this covering. According to $\S 4$, the singular points of $\Xi$ correspond to line bundles:

$$
L=\pi^{*} h^{*} M(x+y)
$$

where:

- $M$ is a line bundle of degree 2 on $E$;

$-\pi x+\pi y \equiv h^{*} p$ where $\mathcal{O}_{E}(p)=\delta \otimes M^{-2}$;

- $h^{0}(L)$ is even.

Such a line bundle gives a vanishing theta-null when $L^{\otimes 2} \simeq \omega_{\tilde{\mathcal{C}}}$, that is:

$$
p=r_{i} \text { and } L=\pi^{*}\left(h^{*} M\left(\tilde{r}_{i}\right)\right) \quad(i=1, \ldots, 10) .
$$

We wish to find such an $L$ with $h^{0}(L)$ even.

Let us fix $i$ and consider the quadratic form $q$ on $\left(\mathrm{JC}_{2}\right.$ associated to the "theta-characteristic" $h^{*} M\left(\tilde{r}_{i}\right)$ (see [M 1] or $\S 1$ ). Notice that $h^{0}(L) \equiv q(\eta)$. If we replace $M$ by $M \otimes \alpha\left(\alpha \in(J E)_{2}\right)$, we get a new line bundle $L^{\prime}$ with:

$$
h^{0}\left(L^{\prime}\right)=q\left(h^{*} \alpha\right)+q\left(h^{*} \alpha \otimes \eta\right)=q(\eta)+\left(h^{*} \alpha \cdot \eta\right)=q(\eta)+(\alpha \cdot N m \eta) \quad(\bmod ) .
$$

Now we distinguish two cases:

$\left.c_{1}\right) \sigma^{*} \eta \neq \eta$. Then $N m \eta \neq 0$, so we can always find a theta-characteristic $L$ with $h^{0}(L)$ even - which gives a vanishing theta-null.

$\left.c_{2}\right) \sigma^{*} \eta=\eta$. Then $\tilde{C}$ is a Galois covering of $E$, with group $\mathbf{Z} / 2 \times \mathbf{Z} / 2$. Such a covering can be constructed in the following way: let $R=r_{1}+\cdots+r_{10}$ be the 
ramification divisor of $h$; we take a partition $R=R_{1}+R_{2}$ with $R_{i} \geqq 0, \operatorname{deg}\left(R_{i}\right)$ even; we choose two line bundles $\delta_{1}, \delta_{2}$ on $E$ such that:

$$
\delta_{i}^{2} \cong \mathcal{O}_{E}\left(R_{i}\right) \quad(i=1,2) \text { and } \delta_{1}+\delta_{2}=\delta .
$$

Let $h_{i}: C_{i} \rightarrow E$ be the associated 2-sheeted coverings, ramified along $R_{i}(i=1,2)$; we put $\tilde{C}=C_{1} \times{ }_{E} C_{2}$, and denote by $\pi_{i}$ the projection $\tilde{C} \rightarrow C_{i}$. The morphism $\tilde{C} \rightarrow E$ factors through $h$ and thus defines a 2-sheeted covering $\pi: \tilde{C} \rightarrow C$.

The line bundle $L$ can be written:

$$
L=\pi_{i}^{*}\left(h_{i}^{*} M(q)\right) \quad \text { where } i=1 \text { or } 2 \text { and } q \in h_{i}^{-1}(p) .
$$

According to Proposition 3.4, one value of $i$ gives an $L$ with $h^{\circ}(L)$ even and the other gives $h^{0}(L)$ odd; we fix $i$ such that $h^{0}(L)$ is even. When $q$ is a ramification point of $h_{i}$, we have $L^{2} \cong \omega_{\tilde{c}}$, so we get a vanishing theta-null. Now $h_{i}$ is always ramified, except possibly when $\operatorname{deg}\left(R_{1}\right)=0, \operatorname{deg}\left(R_{2}\right)=10$. But in that case we find:

$$
\begin{aligned}
& h^{0}\left(\pi_{1}^{*}\left(h_{1}^{*} M(q)\right)\right)=h^{0}\left(h_{1}^{*} M(q)\right)+h^{0}\left(h_{1}^{*}\left(M \otimes \delta_{2}^{-1}\right)(q)\right)=5+0=5 \\
& h^{0}\left(\pi_{2}^{*}\left(h_{2}^{*} M(q)\right)\right)=h^{0}\left(h_{2}^{*} M(q)\right)+h^{0}\left(h_{2}^{*}\left(M \otimes \delta_{1}^{-1}\right)(q)\right)=2+2=4 .
\end{aligned}
$$

Hence, $h_{i}=h_{2}$ is ramified.

(8.1.2) Case $f$. We use the notation of (7.2): so $C$ is obtained from a hyperelliptic curve $N$ by identifying $p_{1}$ to $p_{2}, p_{3}$ to $p_{4}$;

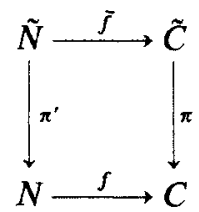

we denote by $H$ the line bundle on $N$ with $\operatorname{deg}(H)=h^{0}(H)=2$, by $\tilde{p}_{i}$ the point of $\tilde{N}$ lying over $p_{i}$. Here again we may assume that $N$ is non-singular. The points of Sing $\Xi$ correspond to line bundles $L$ such that:

$$
f^{*} L=\pi^{*} H\left(\sum \tilde{p}_{i}+x+y\right) \quad \text { with }\left(\pi^{\prime} x+\pi^{\prime} y\right) \in|H| .
$$

In order to get $L^{\otimes 2} \cong \omega_{\tilde{c}}$ we must have

$$
f^{*} L^{\otimes 2} \cong f^{*} \omega_{\tilde{C}} \cong \omega_{\tilde{N}}\left(\sum \tilde{p}_{i}\right),
$$

that is $(2 x+2 y) \epsilon\left|\pi^{\prime *} H\right|$.

This happens in particular when $x+y=\pi^{*} r$ and $r$ is one of the 10 Weierstrass points of $N$. In this case, one has:

$$
\tilde{f}^{*} L \simeq \pi^{\prime *}(H \otimes \delta(r))
$$

where $\delta$ is the line bundle on $N$ associated to the covering $\pi^{\prime}\left(\delta^{2}=\mathscr{O}_{N}\left(\sum p_{i}\right)\right)$. Then we conclude as in (7.2) that $L^{\otimes 2} \cong \omega_{\tilde{\mathrm{C}}}$.

(8.1.3) The proof in cases $\mathrm{h}$ ), i), j) is identical to (8.1.2); details are left to the reader. 
Remarks.

(8.2.1) One can prove by the same method that every Prym variety of dimension $h$ with $\operatorname{dim} \operatorname{Sing} \Theta \geqq h-4$ and which is not a Jacobian has a vanishing theta-null.

(8.2.2) In genus 5, a careful study of the curve Sing $\Xi$ described in Theorem 4.10 gives the following result:

Let $(A, \Theta)$ be a principally polarized abelian varieties of dimension 5 . Then $(A, \Theta)$ is a non-hyperelliptic Jacobian if and only if $(\operatorname{Sing} \Theta) /\{ \pm 1\}$ is isomorphic to a stable plane quintic.

(8.2.3) Here we use the notation of (7.7).

In [A-M], Andreotti and Mayer give a set of equations

$$
f_{\alpha}(z)=0
$$

defining $p^{-1}\left(\bar{J}_{5}\right)$ in the open set $\mathscr{E} \subset H_{5}$ :

$\mathscr{E}=\left\{z \in H_{\mathbf{g}}, \theta(u, z)\right.$ is irreducible $\}$.

From Proposition 8.1, we conclude that the $f_{\alpha}$ 's define $p^{-1}\left(J_{5}-\theta_{\text {nul1 }}\right)$ in the open set $\varphi(z) \neq 0$. The ideal of $p^{-1}\left(J_{5}\right)$ in $\mathscr{E}$ consists of all functions $g$ holomorphic on $\mathscr{E}$ such that for some $r \geqq 0$ :

$$
\mathrm{g}(z) \cdot \varphi^{r}(z)=\sum_{\alpha} a_{\alpha}(z) \cdot f_{\alpha}(z) \quad a_{\alpha} \text { holomorphic in } \mathscr{E} .
$$

\section{References}

[A] Artin, M.: Algebraization of formal moduli I. Papers in honor of K. Kodaira, Univ. of Tokyo Press, 1969

[A'C] A'Campo, N.: Le groupe de monodromie du déploiement des singularités isolées de courbes planes I. Math. Ann. 213, 1-32 (1975)

[A-K] Altman, A. B., Kleiman, S.: Introduction to Grothendieck Duality Theory. Lecture Notes in Math. 146. Berlin-Heidelberg-New York: 1970

[A-M] Andreotti, A., Mayer, A.: On period relations for abelian integrals on algebraic curves. Ann. Scuola Norm. Sup. Pisa 21, 189-238 (1967)

[D-M] Deligne, P., Mumford, D.: The irreducibility of the space of curves of given genus. Publ. I.H.E.S. 36, 75-109 (1969)

[EGA] Grothendieck, A.: Eléments de géométrie algébrique. Publ. I.H.E.S.

[F] Farkas, H.M.: Special divisors and analytic subloci of Teichmüller space. Am. J. Math. 88, 881-901 (1966)

[FGA] Fondements de la géométrie algébrique: Séminaire Bourbaki, t. 14 (1961-62) Exposés n²32 and 236 , by $A$. Grothendieck

[H] Hartshorne, R.: Residues and duality. Lecture Notes in Math. 20. Berlin-Heidelberg-New York: Springer

[Ho] Hoyt, W.: On products and algebraic families of Jacobian varieties. Ann. Math. 77, 415-423 (1963)

[L] Lang, S.: Abelian varieties. New York: Interscience-Wiley 1959

[M 1] Mumford, D.: Theta characteristics of an algebraic curve. Ann. Sci. Ecole Norm. Sup. 4, 181-192 (1971)

[M 2] Mumford, D.: Prym Varieties I. Contributions to analysis. New York: Academic Press 1974

[M 3] Mumford, D.: Abelian varieties. Tata Inst. Studies in Math. Oxford: University Press 1970 
[M 4] Mumford, D.: Geometric invariant theory. Berlin-Göttingen-Heidelberg: Springer 1965

[M 5] Mumford, D.: On the equations defining abelian varieties. Inventiones math, 1, 287-354 (1966)

[O-S] Oda, T., Seshadri, C.S.: Compactifications of generalized Jacobians. To appear

[R] Recillas, S.: Jacobians of curves with $g_{4}^{\mathbf{t}}$ 's are the Prym's of trigonal curves. Bol. de la Soc. Math. Mexicana. 19, 9-13 (1) (1974)

[S] Seshadri, C.S.: Space of unitary vector bundles on a compact Riemann Surface. Ann. Math. 85, 303-336 (1967)

[S-D] Saint-Donat, B.: On Petri's analysis of the linear system of quadrics through a canonical curve. Math. Ann. 206, 157-175 (1973)

[Se] Serre, J.-P.: Groupes algébriques et corps de classes. Paris: Hermann 1959

[Sz] Szpiro, L.: Travaux de Kempf, Kleiman, Laksov. Sém. Bourbaki Exp. 417. Lecture Notes in Math. 317. Berlin-Heidelberg-New York: Springer 1973

[W] Wirtinger, W.: Untersuchungen über Thetafunktionen. Teubner 1895

Received November 5, 1976 